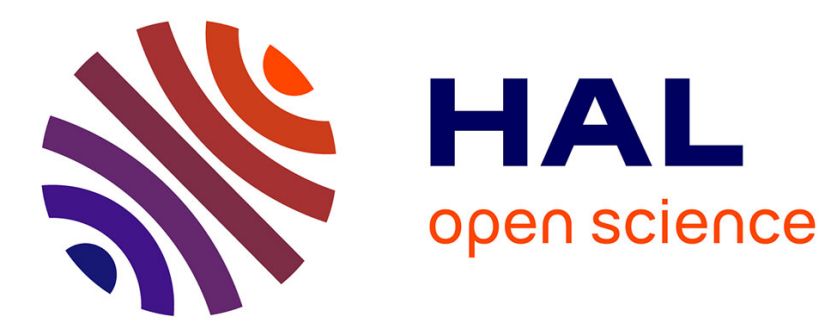

\title{
Poroelastic Relaxation in Thermally Cracked and Fluid-Saturated Glass
}

Abdulwaheed Ògúnsàmì, Jan Borgomano, Jérôme Fortin, Ian Jackson

\section{To cite this version:}

Abdulwaheed Ògúnsàmì, Jan Borgomano, Jérôme Fortin, Ian Jackson. Poroelastic Relaxation in Thermally Cracked and Fluid-Saturated Glass. Journal of Geophysical Research: Solid Earth, 2020, 125 (2), pp.e2019JB018890. 10.1029/2019JB018890 . hal-02567570

\section{HAL Id: hal-02567570 https://hal.science/hal-02567570}

Submitted on 7 May 2020

HAL is a multi-disciplinary open access archive for the deposit and dissemination of scientific research documents, whether they are published or not. The documents may come from teaching and research institutions in France or abroad, or from public or private research centers.
L'archive ouverte pluridisciplinaire HAL, est destinée au dépôt et à la diffusion de documents scientifiques de niveau recherche, publiés ou non, émanant des établissements d'enseignement et de recherche français ou étrangers, des laboratoires publics ou privés. 


\section{RESEARCH ARTICLE \\ 10.1029/2019JB018890}

\section{Special Section: \\ Physical Properties of Rocks, Friction and Fracturing: the \\ Walsh Volume}

\section{Key Points:}

- Broadband bulk, Young's, and shear moduli dispersion and dissipation have been measured on thermally cracked glass specimens, using a combination of forced-oscillation and ultrasonic techniques

- Crack aspect ratios, of mainly $<2 \times$ $10^{-4}$, are inferred from the strong pressure dependence of axial strain, ultrasonic elastic wave speeds, and permeability for differential pressure $P_{\mathrm{d}}<15 \mathrm{MPa}$

- The mechanical properties of the water-saturated specimens show evidence of both draining and squirt transition at the lowest differential pressures $\leq 5 \mathrm{MPa}$

Supporting Information:

- Supporting Information S1

- Data Set S1

Correspondence to:

A. Ògúnsàmì,

abdulwaheed.ogunsami@anu.edu.au

Citation:

Ògúnsàmì, A., Borgomano, J. V. M., Fortin, J., \& Jackson, I. (2020). Poroelastic relaxation in thermally cracked and fluid-saturated glass. Journal of Geophysical Research: Solid Earth, 125, e2019JB018890. https://doi. org/10.1029/2019JB018890

Received 15 OCT 2019 Accepted 19 JAN 2020 Accepted article online 26 JAN 2020

\section{Poroelastic Relaxation in Thermally Cracked and Fluid-Saturated Glass}

\author{
Abdulwaheed Ògúnsàmì̀ $^{1}$ iD, Jan V. M. Borgomano ${ }^{2}$ iD, Jérôme Fortin $^{2}$, and Ian Jackson ${ }^{1}$ iD \\ ${ }^{1}$ Research School of Earth Sciences, Australian National University, Canberra, ACT, Australia, ${ }^{2}$ Laboratoire de Géologie, \\ Ecole Normale Supérieure/CNRS, UMR8538, PSL Research University, Paris, France
}

\begin{abstract}
To test theoretical models of modulus dispersion and dissipation in fluid-saturated rocks, we have investigated the broadband mechanical properties of four thermally cracked glass specimens of simple microstructure with complementary forced-oscillation $(0.004-100 \mathrm{~Hz})$ and ultrasonic techniques ( 1 MHz). Strong pressure dependence of moduli (bulk, Young's, and shear), axial strain, and ultrasonic wave speeds for dry conditions attests to essentially complete crack closure at a confining pressure of $15 \mathrm{MPa}$ - consistent with ambient-pressure crack aspect ratios $\leq 2 \times 10^{-4}$. Oscillation of the confining pressure reveals bulk modulus dispersion and a corresponding dissipation peak, near $0.002 \mathrm{~Hz}$ only at the lowest effective pressure (2.5 $\mathrm{MPa}$ ) - attributed to the transition with increasing frequency from the drained to saturated-isobaric regime. The observations are consistent with Biot-Gassmann's theory, with dispersion and dissipation adequately represented by Zener model. Above the draining frequency, axial forced-oscillation tests show dispersion of Young's modulus and Poisson's ratio, and an associated broad dissipation peak centered near $0.3 \mathrm{~Hz}$, thought to reflect local "squirt" flow and adequately modeled with a continuous distribution of relaxation times over two decades. Observations of Young's and shear moduli dispersion and dissipation from complementary flexural and torsional oscillation measurements for differential pressure $\leq 10 \mathrm{MPa}$ provide supporting evidence of the transition with increasing frequency from the saturated-isobaric to the saturated-isolated regime-also probed by ultrasonic technique. These findings validate predictions from theoretical models of dispersion in cracked media and emphasize need for caution in the seismological application of laboratory ultrasonic data for cracked media.
\end{abstract}

\section{Introduction}

The frequency-dependent nature of seismic properties of fluid-saturated crustal rocks complicates the reconciliation of laboratory ultrasonic measurements of effective moduli (approximately megahertz range) with lower-frequency field data from borehole (approximately tens of kilohertz) and exploration ( 10-100 $\mathrm{Hz}$ ) geophysics. Significant difficulties remain in rigorously testing the theories relating the frequencydependent seismic properties of the fluid-saturated media to microstructures and fluid flow regimes.

In a homogeneous porous medium, dispersion is caused by stress-induced redistribution of fluid within the pore space during wave propagation. Such dispersion and associated strain-energy dissipation reflect the frequency dependence of the spatial scale over which stress-induced gradients in pore pressure can be relaxed by fluid flow. For example, in the low-frequency undrained regime of Biot-Gassmann theory (Biot, 1956a, 1956b; Gassmann, 1951), equivalent to the saturated-isobaric regime of O'Connell and Budiansky (1977), there is sufficient time during the wave period for pore pressure equilibration by fluid flow throughout a representative elementary volume, whereas in the high-frequency unrelaxed or saturated-isolated regime, stress-induced pore pressure gradients between adjacent cracks/pores remain unrelaxed by fluid flow-a condition responsible for higher stiffness/moduli often obtained from laboratory measurements at intermediate to high (ultrasonic) frequencies (e.g., Dvorkin et al., 1995).

Although theoretical models have been proposed to account for the differences between laboratory and field measurements (e.g., Mavko \& Jizba, 1991; O'Connell \& Budiansky, 1977), clear understanding of the mechanism governing the dispersion of elastic properties of crustal rocks remains very topical, particularly as the role of microstructures is yet to be clearly demonstrated by robust and conclusive laboratory investigations. While it is established that the squirt/microscopic (e.g., Mavko \& Jizba, 1991) flow models (which are based on the geometries of the cracks and microstructures) can be efficient in modeling the observed dispersion, the necessary input for this model, that is, quantitative microstructural information such as aspect 
Table 1

The Properties of the Cracked Soda-Lime-Silica-Glass Rods Specimens

\begin{tabular}{lcccc}
\hline Specimen & $\begin{array}{c}\text { Length, mm } \\
( \pm 0.001)\end{array}$ & $\begin{array}{c}\text { Diameter, mm } \\
( \pm 0.001)\end{array}$ & $\begin{array}{c}\text { Crack porosity, } \\
\%( \pm 0.02 \%)\end{array}$ & $\begin{array}{c}\rho_{\text {dry }}, \mathrm{kg} / \mathrm{m}^{3} \\
( \pm 0.09)\end{array}$ \\
\hline FDL2 & 76.200 & 38.100 & 0.74 & 2498.19 \\
SAGR1 & 150.180 & 14.990 & 0.37 & 2507.49 \\
SAGR2 & 150.290 & 15.000 & 0.36 & 2507.75 \\
FDSL1 & 150.010 & 14.980 & 0.30 & 2509.26 \\
\hline
\end{tabular}

Note. FDL2 is the large diameter specimen tested with axial and confining stress oscillation technique. The other specimens were tested with the ANU apparatus. Crack porosities were obtained from dimensional changes after cracking; $\rho_{\text {dry }}$ is the density of the dry specimen. ratios and crack density, is not easily obtained from rocks due to the nature of their complex depositional structures and fabrics. In addition, several of the previous laboratory measurements were made either at low or high frequencies, with insufficient bandwidth to clearly observe the expected transitions between the fluid flow regimes predicted from the theoretical models (e.g., O'Connell \& Budiansky, 1977).

In recent decades, several authors have performed laboratory investigation at both low and high frequencies on selected crustal rocks (e.g., Adam et al., 2006; Adam et al., 2009; Adelinet et al., 2010; Mikhaltsevitch et al., 2014). However, the interpretation of the findings is often compromised by the complex nature of the rock's microstructure. Thermally cracked crystalline rocks and synthetic materials, such as glass, represent alternatives with simpler microstructures but have so far received relatively little attention.

Sarout et al. (2017), for example, tested the seismic properties of thermally cracked marbles, in dry and saturated conditions under effective pressure up to $50 \mathrm{MPa}$, using ultrasonic measurements and low-frequency oscillation of axial stress. They observed Young's modulus dispersion between $0.01 \mathrm{~Hz}$ and $500 \mathrm{MHz}$ in water-saturated conditions with a maximum of $\sim 26 \%$ for $S$ waves and $\sim 9 \%$ for $P$ waves at the lowest effective pressure tested (1 MPa), and the highest crack density ( 0.5). Schijns et al. (2018) tested the seismic properties of dry and fluid-saturated thermally cracked quartzite with $\sim 2 \%$ total porosity between 10 and $150 \mathrm{MPa}$ effective pressures. Ultrasonic and low frequency torsional forced oscillation measurements reveal that the shear modulus increases by more than $70 \%$ for the two specimens from 1 to $1 \mathrm{MHz}$, for water saturation at 20 MPa differential pressure. Chapman, Quintal et al. (2018) investigated moduli dispersion in dry and saturated high porosity (45-47\%) uncracked specimens, made of sintered borosilicate glass. The results of the forced-oscillation test show moduli dispersion and attenuation which were attributed to heterogeneity and compressibility contrast in the sample's frame. Li et al. (2018) in a recent extensive laboratory study, investigated broadband frequency dependent elastic properties of synthetic glass media. The particular advantage of synthetic glass is that its simple microstructure is amenable to a more quantitative interpretation of the observed fluid flow-related dispersion. Although the work of Li et al. (2018) shows evidence of different fluid flow regimes for cracked specimen tested across a range of frequency, no direct evidence of the transitions between the different fluid flow regimes was observed.

Following the study by Li et al. (2018) on synthetic glass media, this contribution brings in new measurements on cracked glass specimens saturated with different pore fluids using different complementary measurement techniques. We report results of newly conducted direct measurements of the dispersion of Young's and bulk moduli and attenuation for a suite of thermally cracked glass rod specimens involving oscillation of either the axial stress or confining pressure. We also present the results of additional torsional and flexural oscillation measurements over a range of $P_{\mathrm{d}}$ (confining-minus-pore) pressure with pentane or deionized water as pore fluid. Together, these results capture a wide frequency interval with clear evidence of modulus dispersion and associated attenuation that we interpret in terms of drainage and squirt flow.

This paper is organized as follows. First, we provide a brief description of the experimental methodology. In the subsequent sections, the results for the specimen characterization, permeability and seismic properties are presented. The observed dispersion and dissipation peaks, together with the microstructure are then interpreted and discussed using rock physics models in the context of the fluid flow regimes in a fluidsaturated cracked medium. Finally, we compare our observations with data from the literature and draw implications for the use of laboratory data in calibrating seismological models for the shallow Earth.

\section{Experimental Methods}

\subsection{Sample Characterization}

Dense soda-lime glass rods, provided by Nadège Desgenétez of the ANU School of Art, were cut and machine ground to obtain cylindrical specimens (see figure in Li et al., 2014). Two groups of specimens (Table 1) were obtained depending on the required dimensions for the applicable testing techniques; 
large diameter (38 mm) for experiments performed at ENS Paris (FDL2) and small diameter (15 mm) for experiments performed at ANU (SAGR1; SAGR2 and FDSL1). Note that the large diameter specimen FDL2 has been previously tested in Li et al. (2018). Using Electron Probe Micro Analysis, the chemical composition of the glass rod is made up of $79.6 \% \mathrm{SiO}_{2}, 12.1 \% \mathrm{Na}_{2} \mathrm{O}, 4.9 \% \mathrm{CaO}, 1.1 \% \mathrm{Al}_{2} \mathrm{O}_{3}$, and $0.9 \%$ $\mathrm{K}_{2} \mathrm{O}$ (Li et al., 2018).

To induce cracks, a heat procedure was applied on the intact rods. We aimed to produce thin cracks that are very compliant (i.e., pressure sensitive). The heat treatment we have applied is as follows. We used an airatmosphere box furnace. The box furnace was first preheated to $500^{\circ} \mathrm{C}$, after which the specimen was placed in it, to dwell for $2 \mathrm{hr}$ and then quenched immediately in water at room temperature $\left(20^{\circ} \mathrm{C}\right)$. Since our initial materials were intact homogeneous rods, crack porosity constitutes the only porosity type in our specimens.

Before thermal cracking, we calculated the volume of each of the specimen from its diameter and length. After the heat treatment, the volume of each specimen was re-calculated. The crack porosities (Table 1) of the specimens consistently obtained as $<1 \%$ were then obtained as the difference in the volume of each specimen, before and after thermal cracking. The density (Table 1$)$ of the dry cracked specimen $\left(\rho_{\text {dry }}\right)$ was determined as

$$
\rho_{d r y}=\left(1-\Phi_{\mathrm{c}}\right) \rho_{s}+\Phi_{c} \rho_{\text {air }}
$$

where $\Phi_{c}, \rho_{s}$, and $\rho_{\text {air }}$ represent the crack porosity, density of the soda-lime glass frame $\left(2515.6 \mathrm{~kg} / \mathrm{m}^{3}, \mathrm{Li}\right.$, 2016), and density of air $\left(1.2 \mathrm{~kg} / \mathrm{m}^{3}\right)$, respectively.

In addition, polished blocks made from the sectioned specimens were examined using light microscopy to provide the microstructural information regarding the crack network.

\subsection{Permeability}

In situ measurement of permeability, prior to mechanical testing, has been performed for the following purposes (1) to guarantee that complete fluid saturation conditions have been achieved (2) to ensure the uniformity of the pore fluid pressure throughout the specimen, (3) to provide a value of permeability, $k$, to be used in the estimation of the characteristic frequency for the drained/undrained transition. In the next section, we discuss the details of sample preparation and jacket type, which applies to both the permeability and mechanical testing.

We measured the permeability of the large diameter specimen, FDL2, using the steady-flow approach at the Laboratoire de Géologie, ENS Paris. We applied different pore fluid pressures on the two sides of the sample (upper and lower) to create a pore pressure gradient $\Delta P / l$, and we monitored the consequent fluid flow. Thus, permeability can be deduced following the equation of Darcy's law:

$$
\frac{q}{A_{s}}=\frac{k \Delta P}{\eta L}
$$

where $q$ is the rate of fluid flow $\left(\mathrm{m}^{3} \mathrm{~s}^{-1}\right), A_{s}$ the sample cross-sectional area $\left(\mathrm{m}^{2}\right), L$ the sample length, and $\eta$ is the dynamic viscosity of the fluid $(\mathrm{Pa} \cdot \mathrm{s})$. We used the standard dynamic viscosity of water, for example, from Venard and Street (1975) and Chemistry WebBook of the National Institute of Standard and Technology (NIST). Permeability was measured at three representative differential pressures, with $P_{\mathrm{d}}<10 \mathrm{MPa}$.

For each of the other three specimens (FDSL1, SAGR1, and SAGR2), the permeability was measured with the transient-flow method (Brace et al., 1968). The measurements were performed at ANU on the attenuation rig with either condensed fluid or compressed gas as pore fluid. The technique involves a sample, enclosed in annealed copper jacket, bounded by two reservoirs initially of equilibrated (equal) pore pressure. A pressure perturbation is then suddenly imposed on one of the reservoirs and the consequent pressure evolution on the unperturbed reservoir is monitored. We applied a sudden pressure increase (decrease) to the reservoir of larger volume. The exponential increase (decrease) with elapsed time of the fluid pressure in the other reservoir, reflecting re-equilibration by fluid flow through the specimen, allows a measurement of the permeability (see details in Li et al., 2018). For argon, we applied the pressure-dependent bulk modulus from Stewart and Jacobsen (1989) and pressure-dependent bulk viscosity from Vidal et al. (1979) as used in Li (2016). We obtained similar information for pentane from Audonnet and Pádua (2001) and Ding et al. 


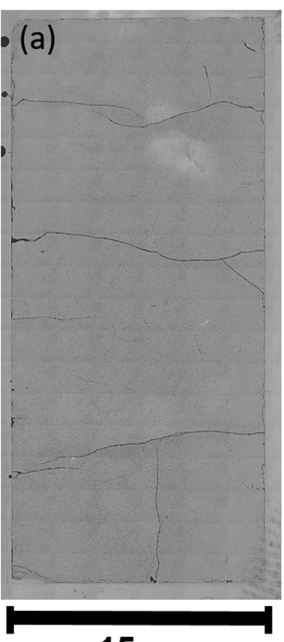

$15 \mathrm{~mm}$

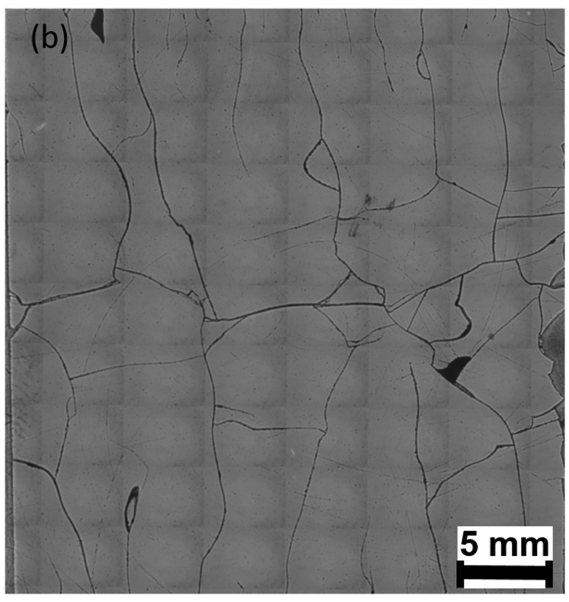

(c)

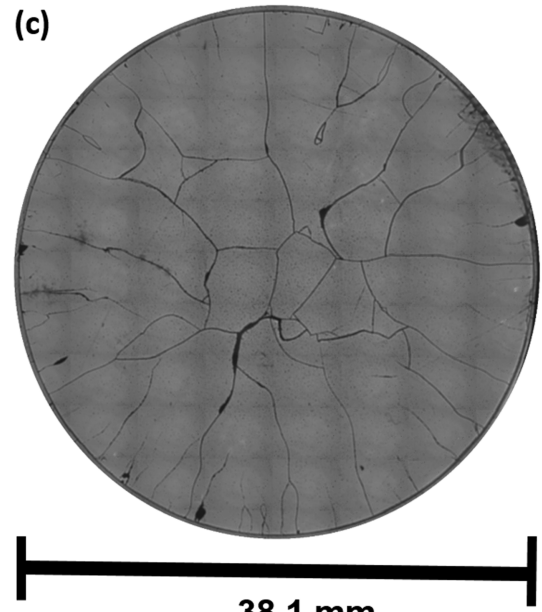

$38.1 \mathrm{~mm}$

Figure 1. Mosaic photomicrographs, representative of the crack microstructure of the specimens: (a) longitudinal section for SAGR1 of 15 mm in diameter; (b) longitudinal section of FDL2 of $38 \mathrm{~mm}$ in diameter; (c) transverse section for FDL2. Dark more equant domains are epoxy-filled cavities resulting from plucking during the preparation of the section.

(1997). For the saturation and fluid charging procedure, we applied the protocol described by Li (2016). This comprised repeated episodes of evacuation of the pore fluid system followed by suction/injection of fluid from an external reservoir until full saturation of the pore space was indicated by a sudden increase of pore pressure during the advance of the intensifier piston. Finally, any gas trapped within the pore fluid reservoirs was bled off before measurements of permeability and seismic properties.

\subsection{Seismic properties}

\subsubsection{Oscillation of Axial Stress or Confining Pressure-E, $v$, and $K$}

Seismic properties of the glass specimen FDL2 of Li et al. (2018) were measured with the low-frequency apparatus at the Laboratoire de Géologie, of ENS Paris. A more detailed description of the setup has been provided elsewhere (e.g., Borgomano et al., 2017; Chapman, Borgomano et al., 2018 and recently in Yin et al., 2019). Here, only a summary of the essential components is provided for completeness. This apparatus allows for (1) hydrostatic pressure using hydraulic oil as the confining medium and (2) independent control and application of pore pressure within the cylindrical specimen. The vertical position of the axial piston can be adjusted to allow the cell to be used for either (a) axial stress oscillation measurement when the piston is directly in contact with the specimen or (b) confining pressure oscillation when the piston is detached from the specimen. The dynamic moduli (Young's and bulk), Poisson's ratio, and associated attenuation are measured by applying a sinusoidally time-varying axial stress or confining pressure to the rock, and measuring the resulting axial and circumferential strains on the rock sample and the axial strain in the reference material (aluminum) (Borgomano et al., 2017; David et al., 2013; Pimienta et al., 2017). This approach has been used for the measurement of Young's modulus by several other authors, for example, Batzle et al. (2006), except that a few components of the setup are slightly different. For example, rather than an electromechanical shaker as used by Batzle et al. (2006), a piezoelectric actuator, mounted between the axial piston of the cell and the top end platen, is used to apply a sinusoidal axial force to the jacketed cylindrical specimen of 76 $\mathrm{mm}$ length and $38 \mathrm{~mm}$ diameter. Note that, when used for a test involving axial stress oscillation, mechanical coupling with the specimen is guaranteed by applying a steady small axial stress $(1 \mathrm{MPa})$ on the assemblage. For the test involving oscillation of the confining pressure (e.g., Adelinet et al., 2010; David et al., 2013; Pimienta et al., 2015), the pressure control system is used to perturb the confining pressure, providing lowamplitude hydrostatic stress oscillation around a mean value of confining pressure. The amplitude of pressure oscillation $\Delta P_{c}$, and the resulting volumetric strain, is sufficiently low that the material's response to stress is linear (Pimienta et al., 2015).

The aluminum end platen, located immediately above the specimen, constitutes the reference material. It is equipped with two axial strain gauges which act as the axial stress sensor (Borgomano et al., 2017; Figure 1). The average of the strains $\left(\varepsilon_{\mathrm{al}, \mathrm{av}}\right)$ obtained from these gauges is used, together with the known Young's 


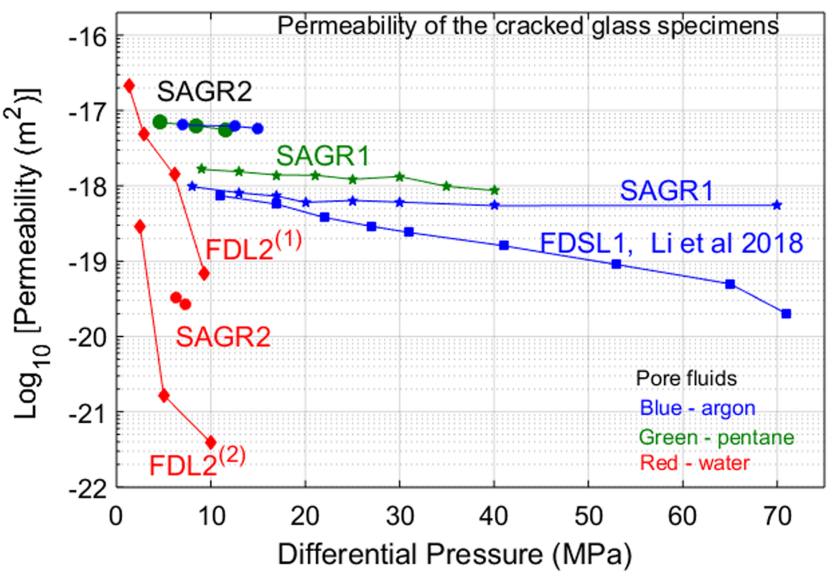

Figure 2. Permeability of the thermally cracked glass specimens as a function of differential pressure. Specimens FDSL1, SAGR1, and SAGR2 were tested with the transient-flow method, and FDL2 with the steady-flow method with tap water during two test campaigns, labeled (1) from Li et al. (2018) and (2) for data obtained in this study. Specimen SAGR2 was tested with deionized water in this study. modulus of aluminum $\left(E_{\mathrm{al}, \mathrm{av}}=72 \mathrm{GPa}\right.$, $)$ to obtain the axial stress, $\sigma_{\mathrm{x}}=E$. $\varepsilon_{\mathrm{al}, \mathrm{av}}$. The axial $\left(\varepsilon_{\mathrm{ax}}\right)$ and circumferential (radial) $\left(\varepsilon_{\mathrm{rad}}\right)$ strains in the test specimen were obtained from the axial and circumferential strain gauges glued directly onto the specimen. Young's modulus $E$ of the specimen is thus obtained as the ratio of the axial stress to the specimen's axial strain. Poisson's ratio $v$ is obtained as the ratio of the radial strain to the axial strain, as in the form below:

$$
E=\frac{\sigma_{X}}{\varepsilon_{a x}} \text { and } \nu=-\frac{\varepsilon_{r a d}}{\varepsilon_{a x}}
$$

The estimated relative uncertainties in the measured Young's modulus $(\delta E / E)$ and Poisson's ratio $(\delta \nu / \nu)$ are around $12 \%$ and $8 \%$, respectively, obtained using the procedure described in Borgomano et al. (2017). As shown, for example, in Yin et al. (2019), the uncertainties depend mainly on the number of strain gauges, two pairs of strain gauges being used in the present study. So for the measurements on the water-saturated conditions at $2.5 \mathrm{MPa}$, for instance, the corresponding error is $\pm 2.9 \mathrm{GPa}$ and 0.03 for $E$ and $\nu$, respectively, with a broadly comparable magnitude at other confining/differential pressures.

For the forced oscillation of the confining pressure, the ratio of the hydrostatic stress to the resulting volumetric strain $\varepsilon_{\mathrm{vol}}=\varepsilon_{\mathrm{ax}}+2 \varepsilon_{\mathrm{rad}}$ provides the bulk modulus $K$ as

$$
K=-\frac{\Delta P_{c}}{\varepsilon_{v o l}}
$$

The estimated relative uncertainty in the measured bulk modulus is $5 \%$, derived from minor uncertainty in the confining pressure $(\delta P / P)$ and significant uncertainties in the strain measurements $(\delta \varepsilon / \varepsilon)$. We obtained the associated dissipation as phase difference between the applied stress and resulting strain $\left(\Delta \phi=\phi_{\text {stress }}-\phi_{\text {strain }}\right)$. So the Young's dissipation from the phase shifts between the axial stress $\sigma_{\mathrm{ax}}$ and strain $\varepsilon_{\mathrm{ax}}$, such that $Q_{E}^{-1}=\tan \left(\phi \sigma_{\mathrm{ax}}-\phi \varepsilon_{\mathrm{ax}}\right)$. The bulk dissipation $\left(Q_{K}^{-1}\right)$ for the hydrostatic oscillation from the phase shifts between the hydrostatic stress $\Delta P_{\mathrm{c}}$ and the volumetric strain $\varepsilon_{\mathrm{vol}}$, such that $Q_{E}^{-1}=\tan$ $\left(\phi_{\Delta P \mathrm{c}}-\phi_{\varepsilon \mathrm{vvol}}\right)$.

The FDL2 specimen was tested in both dry and water-saturated conditions. The specimen was first kept in the oven for $24 \mathrm{hr}$ before being prepared for the measurement. The dry measurements were obtained at 2.5$30 \mathrm{MPa}$ confining pressure $\left(P_{c}\right)$. The fluid saturation protocol involves the injection of tap water for at least $48 \mathrm{hrs}$ at a relatively low differential pressure of $1 \mathrm{MPa}$ (confining pressure at $4 \mathrm{MPa}$, and pore fluid pressure at $3 \mathrm{MPa}$ ), to ensure complete saturation of the low-permeability specimen. After the saturation procedure, a constant pore pressure $\left(P_{\mathrm{f}}\right)$ of $3 \mathrm{MPa}$ was imposed and measurements were taken at differential pressures $\left(P_{\mathrm{d}}=P_{\mathrm{c}}-P_{\mathrm{f}}\right)$ up to $30 \mathrm{MPa}$. At each pressure step during the measurement for the saturated specimen, confining pressure was increased in steps of $2.5 \mathrm{MPa}$, at a controlled rate of $0.01 \mathrm{MPa} / \mathrm{s}$, and kept constant for a period of time for the pore pressure condition to fully equilibrate. At each value of confining (and differential) pressure, the tests involving oscillation of the confining pressure and of axial stress, and ultrasonic wave speed and permeability measurements were then successively performed. The specimen was then dried and prepared for multipath wave speed measurement as described below.

\subsubsection{Torsional and Flexural Oscillations $-G$ and $E$}

Torsional and flexural forced oscillation tests have been conducted on thermally cracked glass specimens in dry and saturated (argon, pentane, and deionized water) conditions. These experiments were performed using the attenuation apparatus at the Australian National University (Li et al., 2018; Figure 2). This apparatus is a gas-medium rig with capacity for high-pressure conditions (up to $200 \mathrm{MPa}$ ), along with independent application and control of pore fluid pressure on a cylindrical specimen of $15 \mathrm{~mm}$ in diameter $\times 150$ mm in length (Jackson \& Paterson, 1993; Lu \& Jackson, 1996). Li et al. (2018) and Cline and Jackson (2016) have a more detailed description of the setup and its recent upgrades. 
Using this apparatus, the seismic properties of the test specimen at $\mathrm{mHz}-\mathrm{Hz}$ are obtained from the analysis of the response of a composite beam to the application of an oscillating torque or bending force. The beam consists of the test specimen that is connected in series to an elastic standard of known mechanical properties. Displacement of the beam associated with its twist or flexure, upon application of torque or bending force, respectively, is measured by two pairs of three-plate capacitance transducers. The distortion of the specimen assembly, containing the specimen of interest along with steel connecting rods, relative to that of the hollow steel elastic element, provides an interim indication of the complex compliance (including the phase lag between applied torque or force and resulting distortion), which is further processed to obtain the modulus and associated dissipation in the specimen itself. To obtain the shear modulus of the test specimen, a similar test on a reference assembly containing an elastic control specimen (uncracked glass) is required. The shear modulus and dissipation $\left(Q_{G}^{-1}\right)$ for the test specimen are computed from the complex compliance differential between the specimen and reference assemblies, along with the known shear moduli for the control specimen and enclosing copper jacket. The uncertainties in the measurements of $3 \%$ in shear modulus and 0.05 $\log$ units in associated dissipation are estimated from a posteriori assessment of scatter among the measured values. To obtain Young's modulus, a filament elongation model is used to extract the optimal Young's modulus of the specimen to match the observed displacements of the cantilevered beam subject to a bending force at the lower end (Cline \& Jackson, 2016; Jackson et al., 2011).

The test specimen is enclosed in an annealed copper jacket of $0.25 \mathrm{~mm}$ wall thickness for isolation from the argon confining pressure medium. The pressure of the pore fluid (argon, pentane, or deionized water, successively), introduced into the crack network of the specimen, taken into consideration along with the confining pressure, enable the test at various differential pressures. Prior pore pressure reequilibration tests ensured conditions of uniform pore pressure throughout the specimen interior.

\subsubsection{Ultrasonic Measurements}

Ultrasonic transducers embedded in each end platen of the axial oscillation set up described in section 2.3.2 were used to study the axial ultrasonic wave speeds in conjunction with the low-frequency oscillation of axial stress or confining pressure. The travel times of the ultrasonic pulses, of $\sim 1 \mathrm{MHz}$ frequency, transmitted through the specimen were processed to obtain the $P$ and $S$ wave speeds (Birch, 1961). For the arrival times and the length of the specimen, the error is $\pm 0.1 \mu \mathrm{s}$ and $\pm 0.01 \mathrm{~mm}$, respectively, while the relative uncertainty on the wave velocity is about $\Delta V / V=0.5 \%$.

To evaluate the possibility of preferred orientation of the thermal cracks in our specimen, we did another ultrasonic experiment in dry condition using another experimental apparatus that has been described in detail in Ougier-Simonin et al. (2011). These experiments enable us to monitor the variation with pressure of the ultrasonic wave speeds by conducting an active multipath ultrasonic survey on the cracked specimen, FDL2. For the multipath survey, we used 10 sources and sensors located along the axis of the specimen. Through perforations in the neoprene jacket used to enclose the specimen, the transducers (lead zirconate, Polytec PI 255) were glued to the specimen's surface. Two pairs of strain gauges, each with the capacity to measure both axial and radial strain, were attached to the specimen. The resonant frequency of the transducers is around $1 \mathrm{MHz}$. An electrical pulse of $250 \mathrm{~V}$ with a rise time of $1 \mu \mathrm{s}$ is generated and applied successively to each of the transducers-generating either $P$ or $S$ waves. The generated waveforms, transmitted through the specimen, were received by the sensors and sampled at $50 \mathrm{MHz}$ for recording by the controlling computer systems.

\section{Results}

\subsection{Microstructures}

Microstructural analysis was performed on the cracked specimens using light microscopy. From each of the specimens, we obtained polished sections to document the characteristics of the crack networks including crack shapes and distribution. We obtained both longitudinal and transverse sections. The large diameter (FDL2) specimen broke into two pieces during the removal of the neoprene jacket after the final test. Only approximately two thirds of the entire length was recovered and was stabilized with epoxy. We took transverse sections at $10 \mathrm{~mm}$ from both ends. We also prepared a vertical plane section of about $40 \mathrm{~mm}$ in length. For the other specimens with $15 \mathrm{~mm}$ diameter (FDSL1, SAGR1, and SAGR2), we took a transverse section right near the top and bottom and also a longitudinal section of approxismately $40 \mathrm{~mm}$. Each of 
the specimens tends to have a unique network of connected cracks. Representative photomicrograph showing details of the crack microstructure is given in Figure 1.

The cracks in our specimens are thin as expected (Figure 1). Estimated average crack aperture is 18 (7) $\mu \mathrm{m}$, while the average crack length for all crack types taken together is 5(2) mm. From these photomicrographs, estimated averaged crack aspect ratios (defined as the ratio of mean crack aperture to mean crack length) is $3 \times 10^{-3}$ (cf. Mallet et al., 2013) and crack density $\sim 0.5$ (defined more formally in equation (6) as $3 \Phi_{c} / 4 \pi \alpha$, where $\Phi_{c}$ is the crack porosity or volume fraction of the cracks and $\alpha$ is the aspect ratio). In the polished sections, the crack shapes are linear to curvilinear, with cracks of different orientation forming a connected network.

Figure 1 shows subsets of cracks with different orientations result from longitudinal, circumferential and terminal radial tensile stresses. The transverse section reveals numerous cracks near the cylindrical surface that tend to coalesce into a smaller number of cracks as they propagate radially inward toward the axis. The longitudinal sections reveal a high density of transverse cracks (e.g., Figure 1a).

\subsection{Permeability}

In previous permeability measurements on cracked glass materials by Ougier-Simonin et al. (2011) and Li et al. (2018), the thermally cracked glass specimens exhibit permeabilities systematically pressure dependent and lower than those typical of tight sandstones (Bourbié et al., 1987).

We measured permeability using both steady- and transient-flow methods. Permeability was more systematically measured at differential pressures $<10 \mathrm{MPa}$ than in Li et al. (2018). In order to address persistent difficulties with water containing various rust inhibitors, permeability measurement was also conducted with deionized water. Argon and pentane, which is broadly comparable to water in viscosity but unlike water nonpolar, were also used as pore fluid.

Figure 2 shows the permeability results for all specimens. The data are best described as defining two distinct broad trends of systematic pressure dependence. For argon and pentane saturation, permeabilities decrease systematically with increasing differential pressure $P_{\mathrm{d}}$ from $\sim 10^{-17.5(5)} \mathrm{m}^{2}$ for $P_{\mathrm{d}}$ near $10 \mathrm{MPa}$ typically to $10^{-18}-10^{-19} \mathrm{~m}^{2}$ but remain measurable by transient flow to $P_{\mathrm{d}}>40 \mathrm{MPa}$. For our measurements with relatively high argon pore pressures (>5 MPa), the flow behavior of the argon is that of a viscous liquid. Accordingly, no Klinkenberg (1941) correction is required (e.g.,Brace et al., 1968; Li, 2016; OugierSimonin et al., 2011; Zoback \& Byerlee, 1975).

In marked contrast, for water saturation, permeability decreases dramatically with increasing $P_{\mathrm{d}}$ to values $\sim 10^{-20(1)} \mathrm{m}^{2}$ near $10 \mathrm{MPa}$, and not measurable with the transient flow technique at higher pressure. For all but the lowest differential pressures, the permeability is much lower with water than with the other fluids (Figure 2). Overall relative uncertainties associated with the permeability measurements are estimated to be $\sim 10 \%$.

\subsection{Hydrostatic Loading-Bulk Modulus and Associated Dissipation}

The results obtained by the oscillation of the confining pressure for specimen FDL2 tested dry and water saturated are presented in Figure 3. The results show that the "dry" bulk modulus increases markedly with increasing confining pressure from 8-10 GPa at the lowest pressure 2.5 MPa to 46-52 GPa for differential pressure $\geq 15 \mathrm{MPa}$ (Figure 3a). Within the low-pressure regime, the bulk modulus increases mildly with increasing frequency in association with modest levels $\left(0.01<Q_{K}^{-1}<0.02\right)$ of strain-energy dissipation. The pressure sensitivity of the bulk modulus is largely suppressed under water-saturated conditions (Figures 3a and 3b) with bulk moduli of 48-52 GPa except at $P_{\mathrm{d}}=2.5 \mathrm{MPa}$ and frequencies $<0.04 \mathrm{~Hz}$. At $P_{\mathrm{d}}=2.5 \mathrm{MPa}$, the bulk modulus decreases and the dissipation increases systematically with decreasing frequency toward the lowest frequency of $0.004 \mathrm{~Hz}$.

The Pseudo-Skempton coefficient $B^{*}$ (Figure 3c), which represents the ratio of the amplitude of the oscillation of pore fluid pressure in the external tubing to the amplitude of the hydrostatic pressure oscillation (Pimienta et al., 2015), shows a significant frequency dependence, particularly for $P_{\mathrm{d}}=2.5 \mathrm{MPa}$. It increases markedly with decreasing frequency especially below $0.02 \mathrm{~Hz}$. 

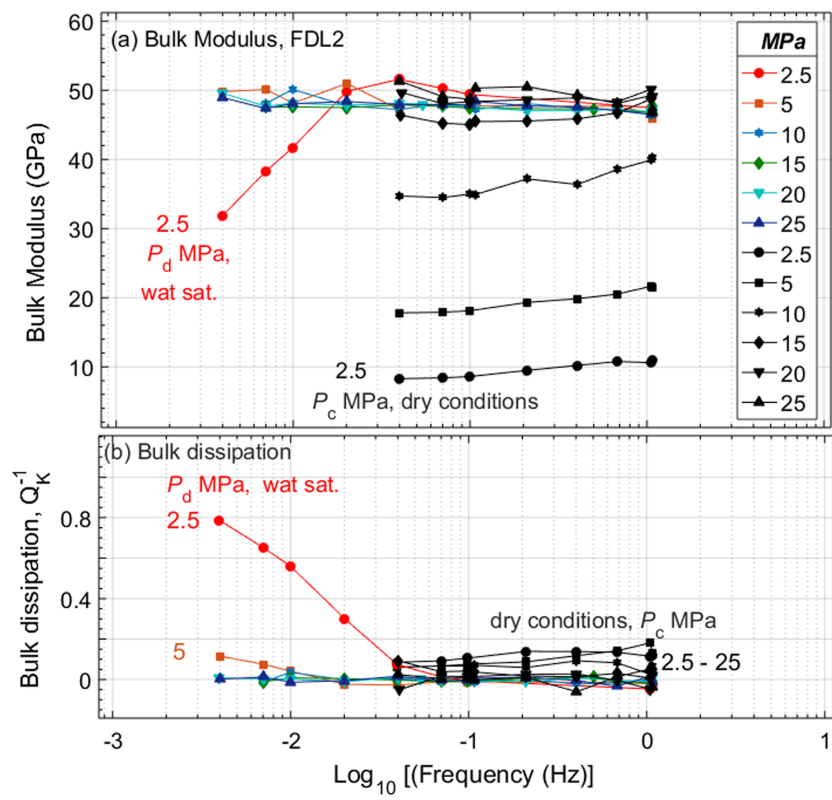

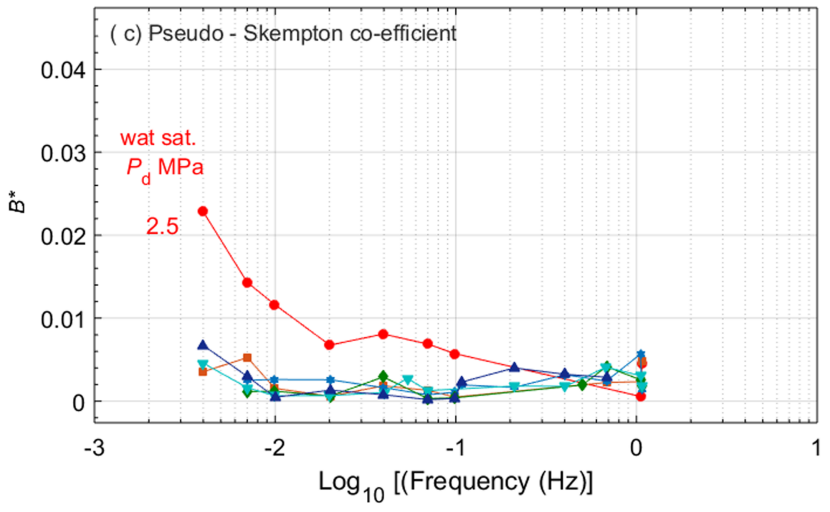

Figure 3. Frequency-dependent behavior of specimen FDL2 tested dry (black symbols) and water-saturated (red to blue symbols) by oscillation of the confining pressure. (a) Bulk modulus $K$; (b) dissipation $Q_{K}^{-1}$; (c) pseudo-Skempton coefficient. Results are obtained for differential pressure in the range of 2.5-25 MPa.

\subsection{Axial Loading-Young's Modulus, Poisson's Ratio, and Associated Strain-Energy Dissipation}

Results of the measurements involving the oscillation of the axial stress are presented in Figure 4. Here, we present Young's modulus and associated dissipation, and Poisson's ratio, as functions of frequency for varied differential pressures to highlight the key observations. Similarly to the bulk modulus, Young's modulus $(E)$ measured under dry conditions increases markedly with increasing confining pressure from 18-24 GPa at $2.5 \mathrm{MPa}$ to $\sim 50 \mathrm{GPa}$ at the highest pressure, $25 \mathrm{MPa}$. The "dry" Young's modulus increases slightly with increasing frequency in association with significant dissipation, well resolved for frequencies $<10 \mathrm{~Hz}$. Such dispersion and dissipation decrease systematically with increasing pressure.
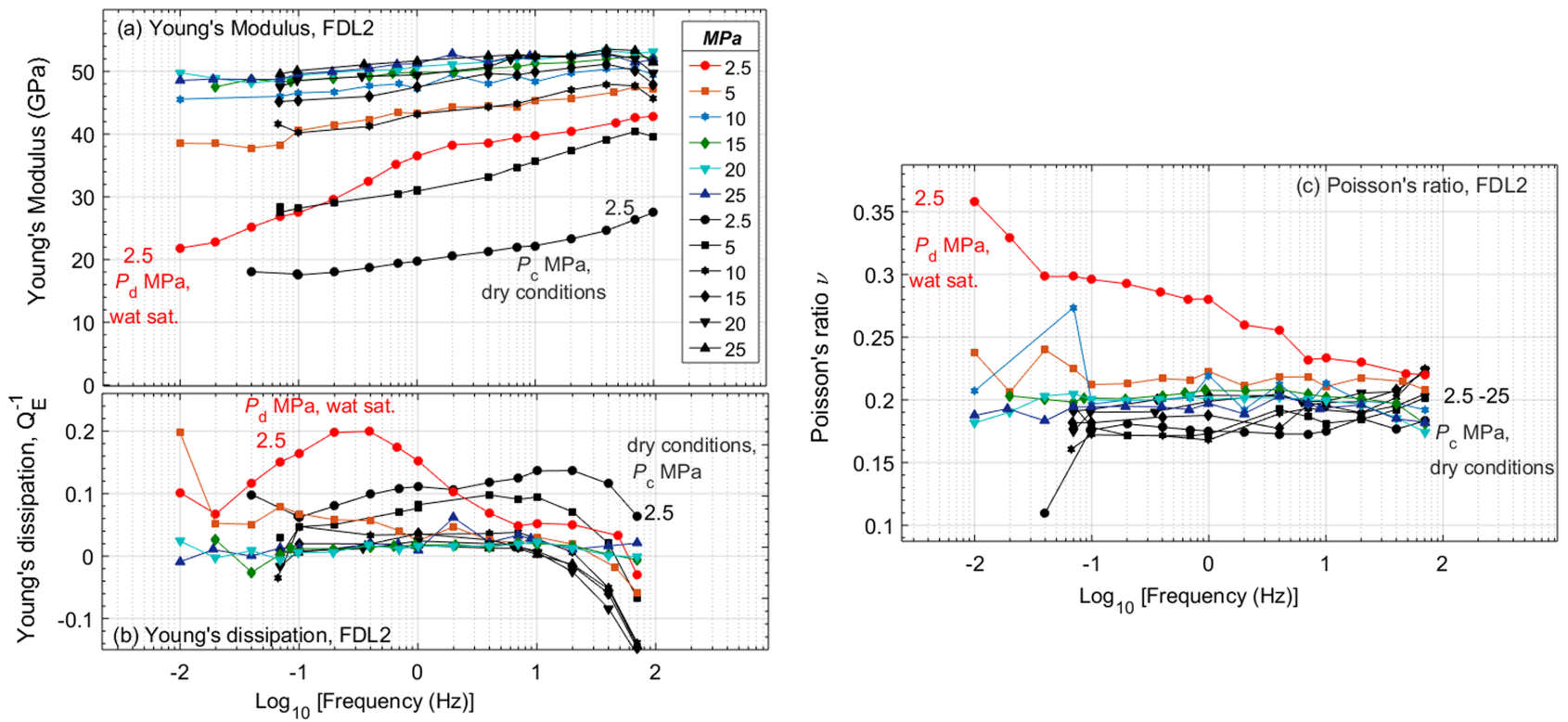

Figure 4. Frequency-dependent (a) Young's modulus, $E$, (b) associated dissipation $Q_{E}^{-1}$, and (c) Poisson's ratio, $\nu$, derived from the axial oscillation experiment for a range of confining and differential pressures, for dry (black symbols) and water-saturated conditions (red to blue symbols), respectively. 

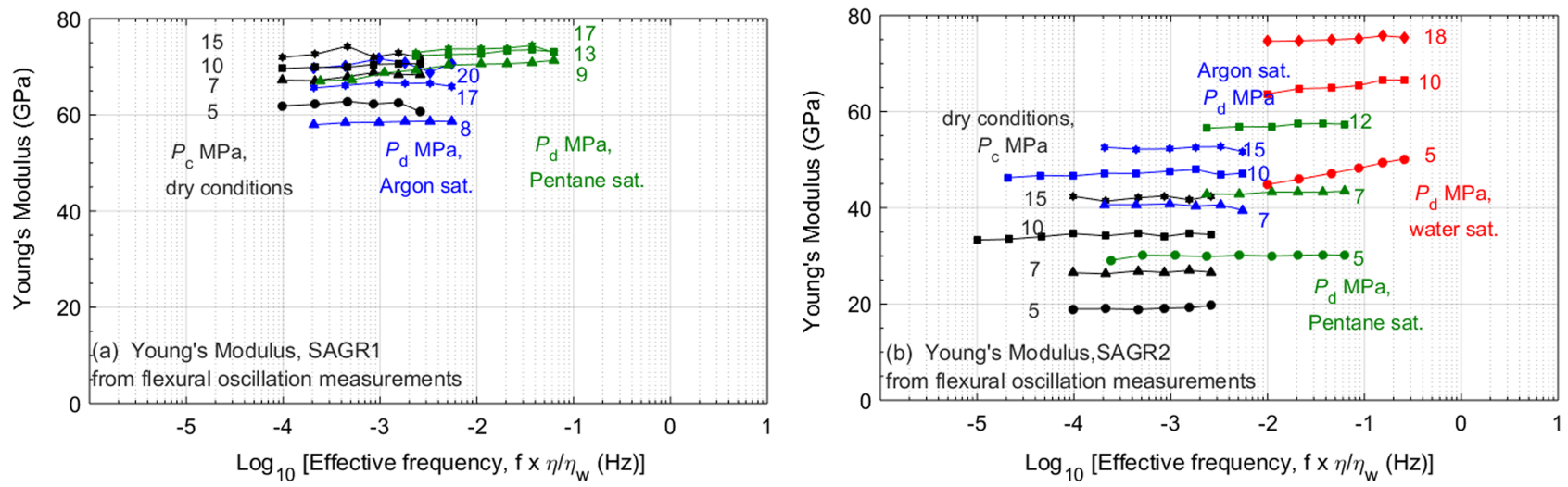

Figure 5. Variation with effective frequency of the Young's modulus for SAGR1 and SAGR2 cracked glass specimens obtained using the ANU apparatus at a range of confining and differential pressures, for dry (black symbols), argon (blue symbols), pentane (green symbols), and water-saturated (red symbols) conditions at a representative range of low confining/differential pressures. The Young's modulus is obtained from flexural oscillation measurements.

Saturation with water results in a marked increase in Young's modulus at low differential pressure (2.5-10 $\mathrm{MPa}$ ). At $P_{\mathrm{d}}=2.5 \mathrm{MPa}$, the "water-saturated" Young's modulus is strongly frequency dependent, especially for frequencies between 0.2 and $0.7 \mathrm{~Hz}$. This regime of strong modulus dispersion at $2.5 \mathrm{MPa}$ coincides in frequency with a strain-energy dissipation peak of substantial amplitude $\left(Q_{E}^{-1} \sim 0.2\right)$. The modulus dispersion and dissipation both decrease markedly with increasing pressure.

The Poisson's ratio of the water-saturated specimen decreases monotonically and strongly with increasing frequency for $P_{\mathrm{d}}=2.5 \mathrm{MPa}$ from $\sim 0.35$ at $0.01 \mathrm{~Hz}$ to $\sim 0.22$ at $70 \mathrm{~Hz}$. With further increase of $P_{\mathrm{d}}$, Poisson's ratio becomes frequency-independent at much lower values approaching those of the dry material.

\subsection{Flexural Oscillation-Young's Modulus and Dissipation}

The flexural forced oscillation data across a range of differential pressure on the dry and fluid-saturated glass-rod specimens SAGR1 and SAGR2 are plotted against effective frequency $f^{*}=\left(\eta / \eta_{\mathrm{w}}\right) f$ in Figure $5 ; \eta$ and $\eta_{\mathrm{w}}$ are respectively the dynamic viscosities of the pore fluid and of water, and $f$ is the experimental oscillation frequency. Use of effective, rather than experimental frequency (in Figures 5 and 6) allows greater clarity in the presentation of the results for saturation with different pore fluids and normalizes the
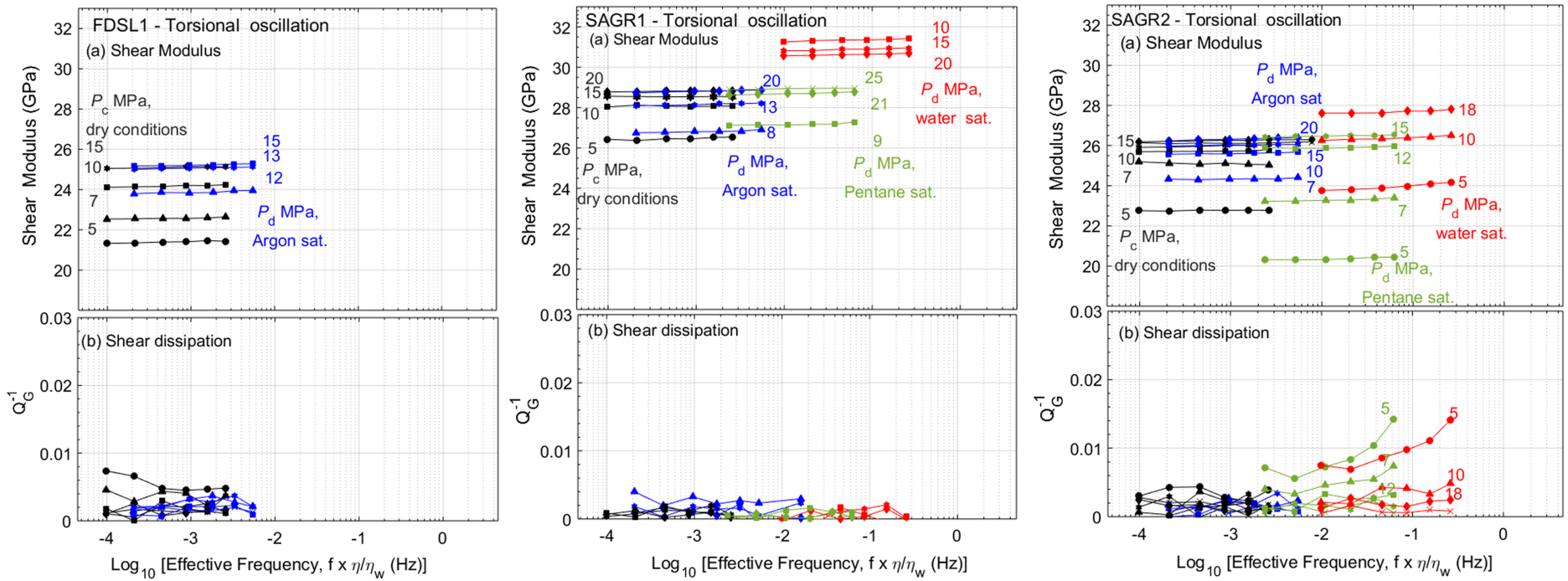

Figure 6. Variation with effective frequency $f^{*}$ of (a) shear modulus, $G$, and (b) shear dissipation $Q_{G}^{-1}$, for FDSL1, SAGR1, and SAGR2 specimens, derived from torsional forced oscillation experiments at a range of confining and differential pressures, for dry, argon, pentane, and water-saturated conditions, respectively. 


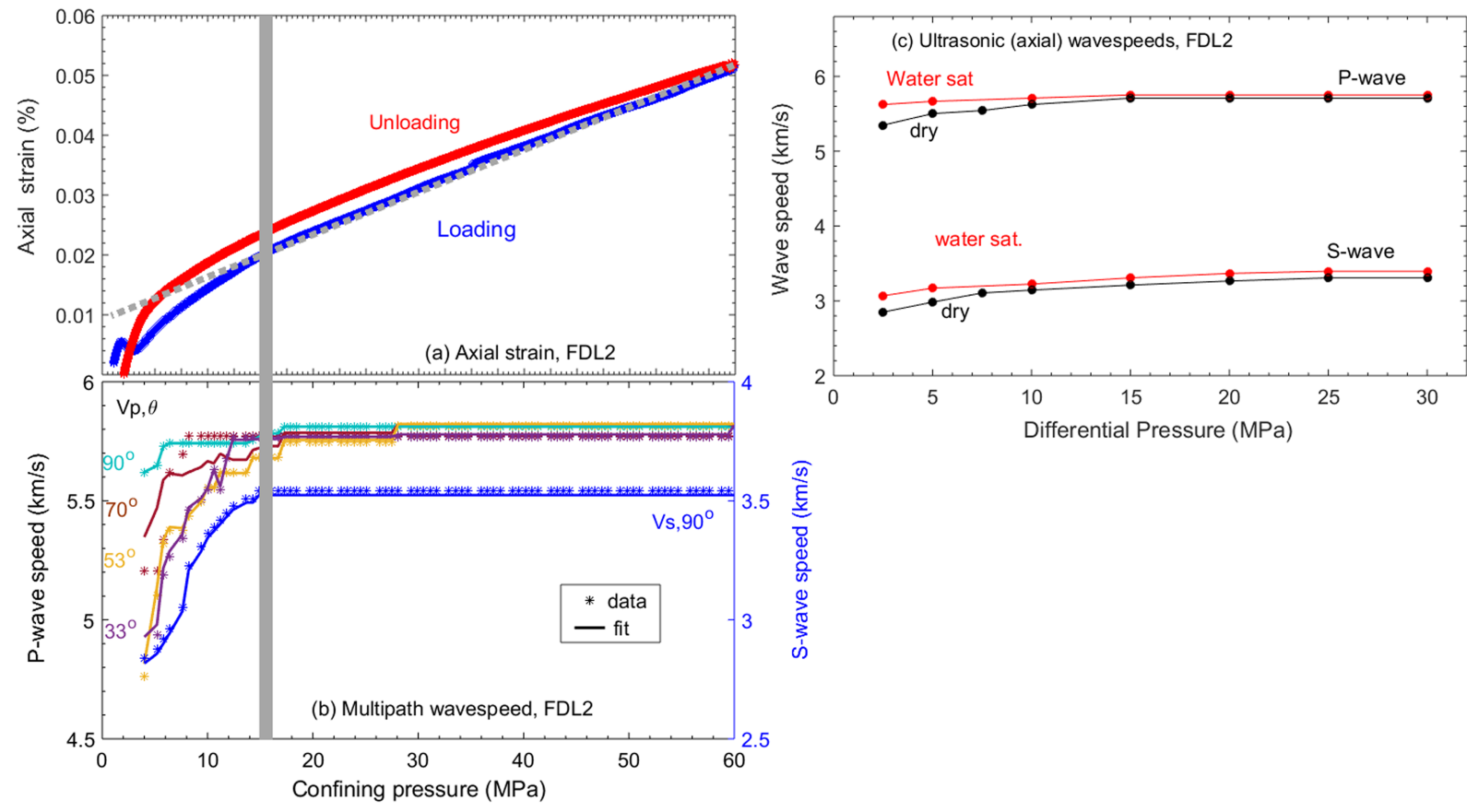

Figure 7. Pressure dependence of (a) axial strain, (b) multipath, and (c) axial ultrasonic wave speeds for the cracked specimen, FDL2. Strain and multipath wave speeds were measured under dry conditions only. Multipath wave speeds (star symbols) were fitted to a transversely isotropic model (see section 4) represented by the curves of different colors representing $V_{\mathrm{P}}$ propagation at various angles $\theta$ of inclination from the cylinder axis (blue for the $\mathrm{SH}$ wave for $\theta=90$ ).

frequencies for the influence of fluid viscosity in anticipation of the discussion related to fluid flow in the next section. Pending the outcome of ongoing improvement of processing routine of the phase lag data for dissipation, we will focus only on the Young's modulus data to provide a general overview of the pressure and frequency dependence.

The Young's modulus increases systematically with increasing confining/differential pressure. Fluid saturation whether by argon, pentane, or deionized water results in a systematic increase Young's modulus. The result for dry, and argon-and (broadly for) pentane-saturated conditions are essentially frequency independent. However, for the water-saturated conditions, a systematic increase of the Young's modulus with increasing frequency is observed-clearest at $P_{\mathrm{d}}=5 \mathrm{MPa}$.

\subsection{Torsional Oscillation-Shear Modulus and Dissipation}

Next, we present data concerning the shear modulus and associated dissipation obtained using the forced torsional oscillation technique (Figure 6).

The shear moduli for the dry conditions are consistently strongly pressure-sensitive for pressures $<15 \mathrm{MPa}$. The shear moduli at the highest pressures vary significantly from one specimen to the other: $\sim 25 \mathrm{MPa}, 29$ $\mathrm{MPa}$, and $26 \mathrm{MPa}$ for FDSL1, SAGR1, and SAGR2, respectively.

Argon and pentane saturation each tends to decrease the shear modulus measured at the lowest values of $P_{\mathrm{d}}$ —clearest for SAGR2 but also evident for SAGR1 and FDSL1. In marked contrast, water saturation systematically increases the shear moduli for both SAGR1 and SAGR2 specimens.

The shear moduli measured under dry and argon-saturated conditions are nearly frequency-independent, and the associated dissipation is typically $<0.005$. On the contrary, frequency-dependent shear modulus associated with substantial dissipation is observed at $P_{\mathrm{d}}<10 \mathrm{MPa}$ for pentane and water-saturated conditions-especially for specimen SAGR2 (Figure 6).

\subsection{Ultrasonic Wave Speeds and Axial Strain}

For the multipath ultrasonic measurements, specimen FDL2 was tested only under dry conditions (Figures 7a and 7b). There are two important observations. First, there is a systematic increase in wave speeds with increasing pressure below $15 \mathrm{MPa}$, beyond which pressure the wave speeds become 
essentially pressure independent. Second, for $P<15 \mathrm{MPa}$, wave speeds vary systematically with propagation direction leading to a compressional wave speed for transverse propagation that is substantially higher than for axial propagation. The axial strain measured in conjunction with the multipath ultrasonic survey increases strongly and nonlinearly with pressure below $15 \mathrm{MPa}$, beyond which a milder linear pressure sensitivity is observed. Comparison of the strain versus pressure trends for loading and unloading reveals a modest hysteresis of the axial stress-strain behavior.

Figure 7c shows the pressure dependence of the ultrasonic wave speeds for axial propagation-measured in conjunction with the axial-loading experiments. The $P$ and $S$ wave speeds show significant systematic pressure sensitivity at $P<15 \mathrm{MPa}$ for dry conditions. The water-saturated conditions show $\mathrm{P}$ - and S-wave speeds that are higher and more mildly pressure dependent than for dry conditions.

\section{Discussion}

\subsection{Insights From Previous Studies of Cracked Glass}

It is well established that the presence of compliant thin cracks strongly affects fluid flow and seismic properties of rocks. The experimental testing, however, of modulus dispersion in cracked media remains inconclusive. Here, we first briefly review a recent study of cracked glass involving measurements of the permeability, elastic moduli, and associated dissipation under dry, argon- or nitrogen-, and water-saturated conditions ( $\mathrm{Li}$ et al., 2018), highlighting the key findings and also some outstanding issues addressed in this study. The elastic moduli, in situ permeability, and crack porosity inferred from in situ X-ray computed tomography indicated strong pressure-induced crack closure for $P_{\mathrm{d}}<30 \mathrm{MPa}$, consistent with zero-pressure crack aspect ratios $<4 \times 10^{-4}$ (cf. Ougier-Simonin et al., 2011). However, in Li et al. (2018) results of the permeability measurements were somewhat inconclusive as the cracked specimens tested by transient flow at differential pressures $\geq 10 \mathrm{MPa}$ proved to be impermeable to water containing rust inhibitors comprising either a suspension of oil and particulate matter or a fully soluble mixture of sodium dichromate and sodium hydroxide. Furthermore, although Li et al. (2018) observed contrasting elastic properties of the saturated-isobaric and saturated-isolated regimes under different experimental conditions, there was no clear observation of any transition between fluid flow regimes. Here we have attempted an intensive sampling of conditions of low differential pressure and extend the frequency range to explore the possibility of observing such transitions. We have also examined the possibility of an anisotropic distribution of crack orientation suggested by the marked difference observed by Li et al. (2018) between axial and circumferential strain below $10 \mathrm{MPa}$.

\subsection{The Behavior of the Dry Cracked Medium}

\subsubsection{Pressure-Dependent Crack Closure}

The observations in this study of strong pressure dependence of all elastic moduli (bulk, Young's, and shear), axial strain, and particularly the multipath ultrasonic wave speeds, consistently restricted to pressures $<15$ $\mathrm{MPa}$, with milder pressure sensitivities for higher pressures, are plausibly attributed to crack closure. The implication from Walsh's (1965) expression $P=E \alpha$ for the crack closure pressure (with Young's modulus $E$ of $75 \mathrm{GPa}$ ) is that most cracks must have aspect ratios $\alpha<2 \times 10^{-4}$. This inference is broadly consistent with that of Li et al. (2018) but with an even narrower distribution of crack aspect ratios. The crack aspect ratios are an order-of-magnitude lower than those estimated from reflected light micrographs (Figure 1) consistent with the idea (Li et al., 2018) that most of the measured crack porosity is contributed by partings of uniform width and effectively zero aspect ratio closed at vanishingly low pressure.

\subsubsection{Transverse Elastic Isotropy of the Dry Cracked Medium}

As noted in the Results section, the ultrasonic compressional wave speeds vary systematically with the inclination $\theta$ of the propagation direction from the cylinder axis for confining pressure $<15 \mathrm{MPa}$ (Figure 7). This observed anisotropy, along with microstructural observations, suggests that the thermal cracks may be preferentially oriented approximately normal to the cylinder axis. The isotropic elastic behavior above $15 \mathrm{MPa}$ is indicative of nearly complete crack closure. The cylindrical symmetry of the stress field responsible for thermal cracking of our glass specimen might reasonably result in such a preferred orientation of cracks, and an associated elastic anisotropy plausibly modeled as transversely isotropic.

We used a first-order perturbation method, to obtain an approximation to the optimal transversely isotropic model. Our method (see details in Appendix A) is based on the assumption that the anisotropy can be adequately modeled as a small perturbation from the isotropic case (e.g., Jech \& Pšenčík, 1989; Ohanian et al., 


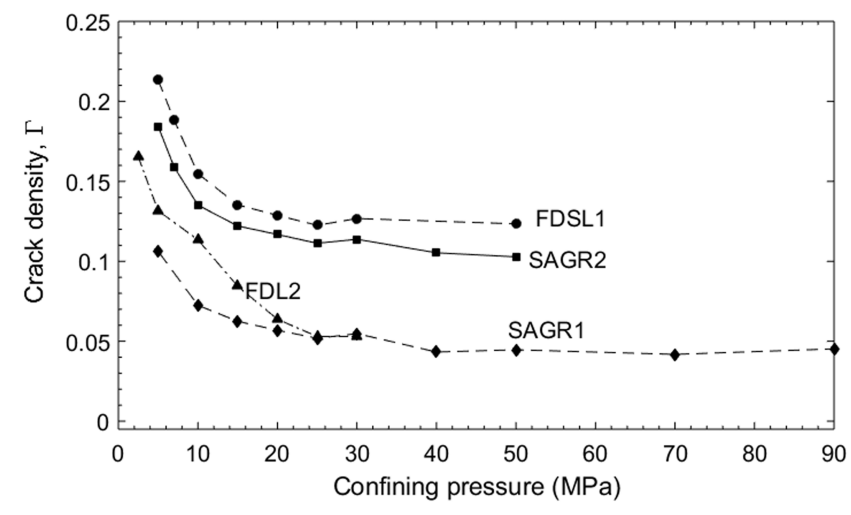

Figure 8. Pressure sensitivity of the crack density obtained using the Walsh perturbation theory from torsional oscillation measurement under dry conditions for FDSL1, SAGR1, and SAGR2 and from ultrasonic shear wave speeds for FDL2
2006) - an approach well known for satisfactory approximation of the elastic constants (e.g., Mensch \& Rasolofosaon, 1997; Song \& Koch, 2002; Wu et al., 2017). Such a method involves the use of a linearized procedure starting from the properties of an isotropic medium (the uncracked glass). The multipath wave speed data $\left[V_{\mathrm{QP}}(\theta)\right.$ for $\theta=33$, 53,70 and $90^{\circ}$ and $V_{\mathrm{SH}}(\theta)$ for $\theta=90^{\circ}$ ] at $P_{\mathrm{c}}=0-60 \mathrm{MPa}$ are adequately approximated by the resulting transversely isotropic model (Figure 7b).

4.2.3. Influence of Transverse Elastic Isotropy on the Moduli Measured at Low Frequencies in Axial and Hydrostatic Loading In Appendix B, we provide a derivation in terms of the $C_{\mathrm{ij}}$ of the effective elastic moduli that govern the response of a transversely isotropic medium to axial and hydrostatic loading. For such a medium, it is shown that Young's modulus and Poisson's ratio derived from axial loading of the transversely isotropic medium cannot be combined, as for isotropic material, to obtain a bulk modulus that is directly comparable with that measured with hydrostatic loading. The assumption of isotropic elasticity is appropriate for differential pressure $>15 \mathrm{MPa}$.

4.2.4. Crack Density Inferred From Effective Medium Model

Following the approach of Walsh (1965), the effective shear modulus $(G)$ of a cracked medium with a dilute concentration of randomly oriented crack inclusions can be expressed in the form (Zimmerman, 1991)

$$
\frac{G_{r}}{G}=1+\frac{32\left(1-v_{r}\right)\left(5-v_{r}\right)}{45\left(2-v_{r}\right)} \Gamma
$$

where $G_{r}$ and $\nu_{r}$ represent the bulk modulus, shear modulus, and Poisson's ratio, respectively, of the uncracked medium. $\Gamma$ is the crack density, defined as

$$
\Gamma=N a^{3}=\frac{3 \Phi_{c}}{4 \pi \alpha}
$$

$N$ represents the number of cracks per unit volume; " $a$ " is the crack radius. $\Phi_{c}$ is the crack porosity or volume fraction of the cracks, while $\alpha$, is the aspect ratio (minimum/maximum dimension). Ultrasonic measurements of elastic properties of uncracked cylinders of the same soda-lime-silica glass (Li, 2016) gives the shear, Young's, and bulk moduli, and Poisson's ratio of uncracked glass rod as $31 \mathrm{GPa}, 75 \mathrm{GPa}, 44 \mathrm{GPa}$, and 0.22 , respectively.

Whereas dry Young's modulus derived using the ultrasonic multipath wave speeds (Figure 8) at high pressure $\left(P_{\mathrm{c}}>15 \mathrm{MPa}\right)$ show values consistent with ultrasonic-frequency Young's modulus for the uncracked glass specimen in Li et al. (2018), the results of the axial oscillation measurement on FDL2 specimen show substantial Young's modulus deficit of at least 20\% remaining at the highest pressure (25 MPa) (Figure 4). For the small-diameter specimens tested in torsion, maximum values of shear modulus as low as $\sim 26 \mathrm{GPa}$ were observed at the highest pressure (Figure 6). Such low shear modulus indicates that a substantial deficit of about 6-16\% remains when compared with $31 \mathrm{GPa}$ for uncracked equivalent measured at an ultrasonic frequency in Li (2016). These observations imply that the pressure-induced closure of thermal cracks can be greatly variable among our cracked specimens.

The crack density calculated from the pressure-dependent shear modulus deficit for each of the specimens is presented in Figure 8. For the small diameter specimens-FDSL1, SAGR1, and SAGR2, the shear modulus obtained from the torsional oscillation measurement at dry conditions was used. However, for the FDL2 specimen the high-frequency shear modulus derived from the axial ultrasonic shear wave speeds was used.

The crack density, $\Gamma$, for the small diameter specimens-FDSL1, SAGR1, and SAGR2-is inferred to decrease from $\sim 0.2,0.1$, and 0.2 at the lowest confining pressures $\left(P_{\mathrm{c}}=5-7 \mathrm{MPa}\right)$ to $0.05-0.1$, at high pressure. The pressure sensitivity of the inferred crack density decreases with increasing confining pressure for all the three specimens. In absolute magnitude and to the extent of its pressure sensitivity, modeled crack density for the FDSL1 and SAGR2 is markedly different from that of SAGR1. The crack density for FDSL1 and SAGR2 is higher by $\sim 0.08$ throughout the measurement range, with the pressure sensitivity 
dominantly below $P_{\mathrm{c}}=20 \mathrm{MPa}$ (Figure 8). The crack density for FDL2 decreases markedly with pressure from $\sim 0.17$ to 0.05 , between 4 and $15 \mathrm{MPa}$ confining pressure.

Here we have assumed homogenous distribution of the cracks and have only used the shear modulus, calculated from the axial shear wave velocity that was measured in conjunction with the axial stress oscillation tests. Note that the overall crack orientation distribution for these cracked specimens may be anisotropic, as informed by the result from the multipath wave speed measurement. Although an extension of the above analysis to the anisotropic appropriate symmetry (Sayers \& Kachanov, 1995) may seem more appropriate, the calculation above serves to illustrate the broad variability of the pressure dependence of the crack density for our specimens.

4.2.5. Frequency Dependence of Moduli and Dissipation Under "Dry" Conditions at Low $\boldsymbol{P}_{\mathrm{c}}$

A significant level of dispersion and associated dissipation are observed even for dry conditions at $2.5 \leq$ $P_{\mathrm{c}} \leq 10 \mathrm{MPa}$ (Figures 3 and 4). Such dispersion and dissipation ( $K, G$, and $E$ ) associated with the "dry" condition are possibly due to the presence of adsorbed moisture in the crack microstructure (e.g., Mavko \& Nur, 1979). At high relative humidity, condensation of water is favored in low aspect ratio cracks or fine capillaries (Tittmann et al., 1980) typical of our cracked specimens. Such very small amount of adsorbed water has been shown (e.g., in Clark et al., 1980; Murphy et al., 1986; Winkler \& Nur, 1982) to significantly affect moduli, and increase dissipation in several sedimentary rock specimens tested in the laboratory. The effect of such moisture adsorbed in thin layers at asperities will be frequency dependent-potentially stiffening the modulus at sufficiently high frequencies and producing dispersion and dissipation at the characteristic frequency for stress-induced deformation of the fluid layer.

\subsection{The Behavior of the Saturated Cracked Medium}

4.3.1. Permeability

Brace et al. (1968) showed that gas and water permeabilities are essentially equal in intact Westerly granite ( $<1 \%$ porosity) over a range of confining pressures between 10 and $100 \mathrm{MPa}$. Ougier-Simonin et al. (2011) showed that the variation of permeability with differential pressure for cracked borosilicate glass is reversible and broadly similar for water and argon pore fluids. However, as previously mentioned, Li et al. (2018) using the transient-flow approach at $P_{\mathrm{d}} \geq 10 \mathrm{MPa}$ found that cracked glass-rod specimens were permeable to argon, but not to water containing alternative rust inhibitors. Even with a fully water-soluble mixture of 0.02 wt. \% sodium dichromate $\left(\mathrm{Na}_{2} \mathrm{Cr}_{2} \mathrm{O}_{7}\right)$ and 0.003 wt. \% sodium hydroxide $(\mathrm{NaOH})(\mathrm{pH}=6.5)$, the cracked glass materials proved to be effectively impermeable to water for $P_{\mathrm{d}} \geq 10 \mathrm{MPa}$ ( $\mathrm{Li}$ et al., 2018; and the present study).

Accordingly, we must consider the alternative whereby the impermeability to water might reflect chemical, rather than purely hydraulic, interaction between the siliceous surface of the narrow crack and the polar water molecule. To address this question we have tested the specimen with pentane-a nonpolar fluid, broadly comparable with water in incompressibility and viscosity. Our result (Figure 2) establishes that specimen SAGR1 is similarly permeable with pentane and argon pore fluids—providing further indication that chemical properties of the pore fluid may control fluid flow through the cracked glass specimens. Heap et al. (2018) measured the permeability of volcanic materials with gas and water as pore fluids, showing that permeabilities to argon gas and deionized water can differ twofold to fivefold in two volcanic rocks (basalt and andesite) over a confining pressure range from 2 to $50 \mathrm{MPa}$. They attributed the difference to water adsorption on the surface of the pore crack network. Such adsorption has been speculated to have reduced the effective pore throat of the thin tortuous/rough microcracks and therefore restricted the access for water. It seems likely that the impermeability of the cracked glass-rod specimens to water-rust inhibitor solution observed by Li et al. (2018) and in the present study is related to chemical interaction between water and the siliceous surfaces of the very narrow cracks.

A useful measure of the strength of the chemical interaction between water and a siliceous glass surface is the capillary pressure, $P_{\text {cap }}$ (Guéguen \& Palciauskas, 1994), given by the Young-Laplace equation as

$$
P_{c a p, H_{2} O}=\frac{2 \cdot \gamma_{a i r, H_{2} O} \cdot \cos \theta}{r}
$$


$\gamma_{\text {air }, \mathrm{H}_{2} \mathrm{O}}$ is the interfacial tension between $\mathrm{H}_{2} \mathrm{O}$ and air, $\theta$ is the contact angle for the aqueous phase on the surface of the glass medium, and $r$ is the characteristic radius of the pores/cracks controlling the percolation of the pore fluid, $\mathrm{H}_{2} \mathrm{O}$. With $\theta=0^{\circ}$ (water wets glass), $\gamma_{\text {air }, \mathrm{H}_{2} \mathrm{O}}=72 \mathrm{mN} \mathrm{m}^{-1}$ (Guéguen \& Palciauskas, 1994) and $r$ of order $1 \mu \mathrm{m}, P_{c, H_{2} O} \sim 140 \mathrm{kPa}$ sufficient to support a meniscus of height $14 \mathrm{~m}$. Capillary pressure related to surface tension may therefore strongly aid the imbibition of water. Similarly, strong chemical interaction must be responsible for restricting the redistribution of water in response to stress-induced gradients in pore pressure.

Thus, although permeability $k$ is usually regarded as a property of the cracked medium and viscosity $\eta$ as a property of the fluid, what is actually measured (on assumption of Darcy proportionality between flux and pressure gradient) is the ratio of $k / \eta$. In this study, water saturation results in systematically lower values of $k / \eta$ than for argon or pentane saturation-reported in Figure 2 as lower values of permeability-perhaps more appropriately regarded as an effective permeability (based on the assumption of normal $\eta$ ). Alternatively, the lower values of $k / \eta$ could be explained in terms of the standard (argon-pentane) permeability and an increased effective viscosity. Our suggestion that the driving force supplied by the prevailing pressure gradient is opposed by the chemical interaction between the polar water molecules and the siliceous surfaces of the narrow cracks thus calls into question the Darcy proportionality between fluid flux and pressure gradient with standard measures of permeability and viscosity.

That said, the higher permeabilities measured with argon and pentane as pore fluids probably include a contribution from bypass flow along the interface between the cylindrical specimen and enclosing annealed copper jacket, evidently precluded for water saturation. Unavoidable scratches on the inner surface of the copper jacket and microcracking of the near-surface region of the glass specimen during precision grinding provide potential conduits for bypass flow. The relatively low permeability measured on specimen FDL2 during the test involving the oscillation of axial stress or confining pressure may be related to the delivery of fluid from a central point source. In contrast, for the measurements made on FDL2 (Li et al., 2018) in the Lawrence Berkeley Laboratory and those on SAGR2 in the ANU laboratory involving higher permeabilities, fluid was distributed over the cross section providing ready access to relatively widely spaced cracks intersecting the end of the specimen.

\subsubsection{The Sensitivity of Seismic Properties to the Nature of the Pore Fluid}

The fluid sensitivity of the low-frequency mechanical properties for our cracked specimens tends to be variable for the different fluid types as summarized in the Figures 3-6. For argon-saturated conditions, the measured moduli are broadly comparable with those of dry conditions (Figures 5 and 6), although there are two exceptions. There is a slight reduction of shear modulus with argon saturation at the lowest values of differential pressure (Figure 6), and an apparently significant increase in Young's modulus on argon saturation of SAGR2 (Figure 5).

For pentane-saturated conditions, somewhat different behavior is observed-especially for SAGR2. The Young's modulus for pentane-saturated SAGR2 is systematically higher than for the dry conditions. On the other hand, the shear modulus $G$ for pentane-saturated conditions is systematically lower than for dry conditions. At the lowest values of $P_{\mathrm{d}}$, the significant positive frequency dependence of $G$ is associated with substantial levels of dissipation $\left(0.005 \leq Q_{G}^{-1} \leq 0.02\right.$; Figure 6). Whereas reduction in shear modulus upon water saturation has been observed by many researchers (e.g., Adam et al., 2006; Khazanehdari \& Sothcott, 2003; Spencer, 1981; Wong \& Baud, 2008; Yin et al., 2019), weakening with nonpolar fluid is scarce in the literature. Published water weakening of the shear modulus has been attributed to two main mechanisms: the chemical influence of pore fluids causing a reduction of the surface free energy (Yin et al., 2019) and the mechanical role of pressurized pore fluid, which tends to modify the grain contacts and therefore the elastic properties (e.g., Tutuncu \& Sharma, 1992). Pentane is nonpolar. Therefore, the reduction of surface energy due to fluid-solid interaction is less likely the explanation. Further work with pentane as pore fluid on our glass specimens will provide for an improved explanation for such weakening effect.

Only for water-saturated conditions are all of the elastic moduli (bulk, Young's, and shear) and the axial ultrasonic velocities systematically higher than for dry conditions (Figures 3-7c). Broadly consistent behavior is observed for tap water, deionized water, and water containing the soluble rust inhibitor. Higher bulk and Young's moduli are to be expected in the presence of an incompressible pore fluid such as pentane or 
water-under undrained conditions. On the other hand, the shear stiffening, observed for water but not pentane saturation requires access to saturated-isolated conditions. The following estimation of the characteristic frequencies for global and local fluid flow, along with a discussion of the modulus dispersion and associated dissipation, will provide the basis for more robust interpretation in terms of fluid flow regimes.

\subsubsection{Fluid Flow Regimes}

The effective elastic moduli of a fluid-saturated cracked medium are expected to depend on the frequency at which they are measured. A series of regimes is identified-each regime defined by the spatial scale on which stress-induced gradients in pore pressure can be relaxed by fluid flow. With increasing frequency, the drained, saturated-isobaric (undrained), and saturated-isolated (unrelaxed) regimes are successively expected (e.g., Cleary, 1978; Gassmann, 1951; Mavko \& Nur, 1975; O'Connell \& Budiansky, 1977—see Li et al., 2018, Figure 1).

Under drained conditions, the stress-induced perturbation in pore pressure within the pore space of the specimen can be fully relaxed by the fluid flow between the interior of the specimen and an external reservoir of sufficient volume. Accordingly, the bulk and shear moduli are unaffected by the presence of the fluid, and the specimen behaves as if it were dry. Under saturated-isobaric (undrained) conditions, it is envisaged that fluid flow allows pore pressure equilibration between differentially pressurized parts of the pore space (of different orientation relative to the applied stress field and/or different aspect ratio) but without an exchange of fluid with an external reservoir. The Biot-Gassmann equations of poroelasticity relate the bulk $K_{\mathrm{u}}$ and shear moduli $G_{\mathrm{u}}$ for the undrained regime to those of the drained conditions $\left(K_{\mathrm{d}}\right.$ and $\left.G_{\mathrm{d}}\right)$, in terms of specimen porosity $\Phi$, the bulk modulus of the fluid $\left(K_{f}\right)$ and of the matrix $\left(K_{m}\right)$ in the form below

$$
\begin{gathered}
K_{u}=K_{d}+\frac{\left(1-\frac{K_{d}}{K_{m}}\right)^{2} K_{f}}{\Phi+\left(\left(1-\frac{K_{d}}{K_{m}}\right)-\Phi\right) \frac{K_{f}}{K_{m}}} \\
G_{\mathrm{u}}=G_{\mathrm{d}}
\end{gathered}
$$

At still higher frequencies of stress application, there will be insufficient time for fluid flow to allow pressure reequilibration between differentially pressurized parts of the pore space such as adjacent cracks of different orientation, or adjacent cracks and pores. Accordingly, this regime is appropriately termed saturated isolated (O'Connell \& Budiansky, 1977) or unrelaxed (Guéguen \& Kachanov, 2011). Relative to the saturated-isobaric regime, saturated-isolated conditions will involve a higher shear modulus as a consequence of the incompressibility of the fluid, and for a distribution of crack/pore aspect ratios, also a higher bulk modulus.

These fluid flow regimes (O'Connell \& Budiansky, 1977) are separated by characteristic frequencies-drainage frequency $f_{\mathrm{dr}}$, demarcating drained conditions from the saturated-isobaric regime, and squirt frequency $f_{\text {sq }}$, which separates the saturated-isobaric and the saturated-isolated regimes. Based on the work of Cleary (1978) and Zimmerman (1991), Li et al. (2018) suggested a modified expression for the drainage frequency,

$$
f_{\mathrm{dr}}=\frac{3 k G}{8(1-v) \Gamma \eta l^{2}}
$$

for a cracked medium saturated with condensed pore fluid, for which the compressibility of the pore space exceeds the contribution of the pore fluid compressibility to the total pore compressibility. In the modified expression, $k$ is permeability of the specimen, $v$ is the Poisson's ratio, $G$ the shear modulus of the specimen, $\Gamma$ is crack density, and $l$ is the length scale over which pore fluid diffusion occurs. Similarly, the squirt frequency can be written as

$$
f_{\mathrm{sq}}=\frac{K \alpha^{3}}{2 \pi \eta}
$$

where $K$ is the bulk modulus of the medium, $\alpha$ is crack aspect ratio, and $\eta$ is the dynamic viscosity.

The fluid-saturated glass specimens in this study show a variability in their mechanical properties-broadly similar to those of Li et al., 2018 at higher differential pressures, but with key differences at low differential 


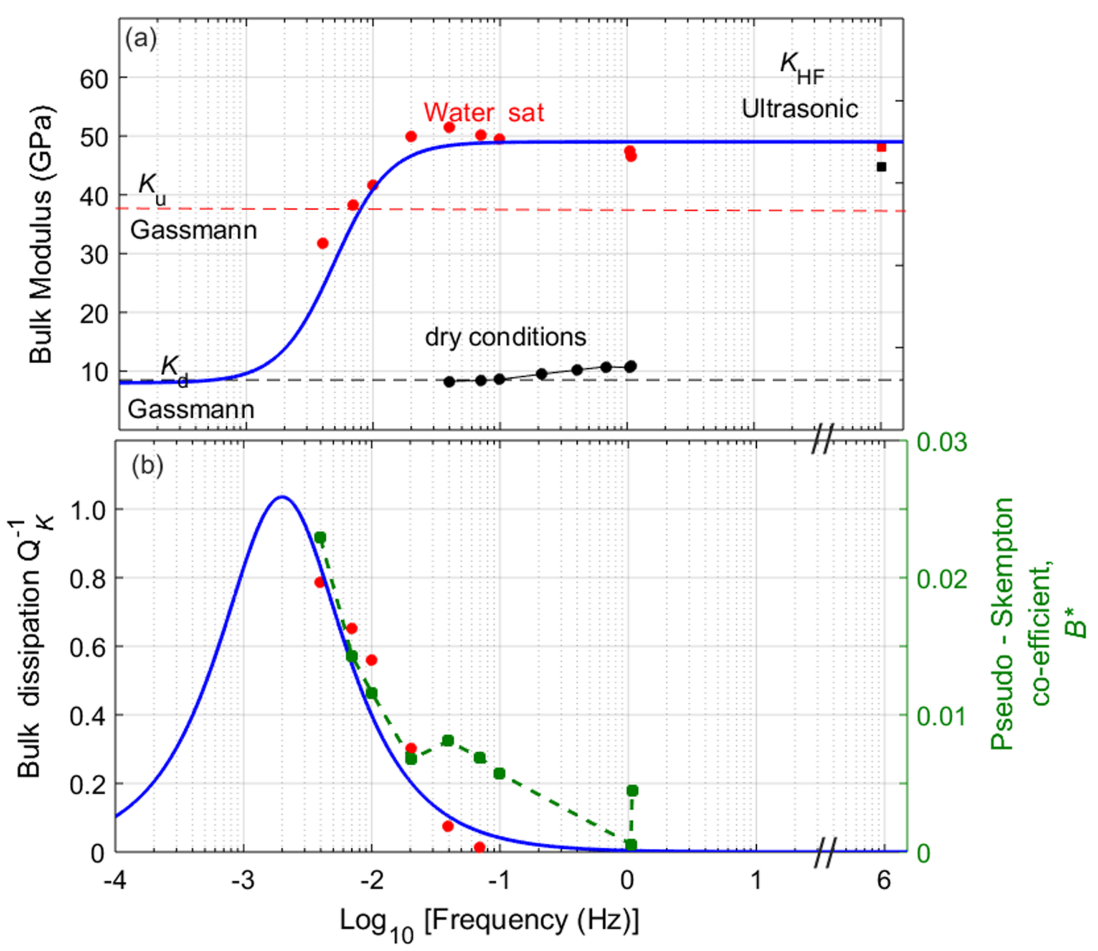

Figure 9. Frequency-dependent (a) bulk modulus, (b) bulk dissipation, and pseudo-skempton coefficient obtained from the test involving the forced oscillation of the confining pressure for the water-saturated FDL2 specimen. Red, black, and green symbols indicate the data points, whereas the blue curve represent model (Biot-Gassmann for modulus, and Zener rheological model for dissipation) fitted to the observed dispersion and dissipation peak.

pressures, $P_{\mathrm{d}}<10 \mathrm{MPa}$. Dispersion of the bulk modulus and associated dissipation newly observed at $P_{\mathrm{d}}=$ $2.5 \mathrm{MPa}$ in the test involving the confining pressure oscillation may signal the transition with frequency increasing beyond $0.001 \mathrm{~Hz}$-from drained to saturated-isobaric (undrained) conditions.

Results obtained at the lowest differential pressure (2.5 MPa) from the oscillation of axial stress for watersaturated conditions reveal dispersion of the Young's modulus and associated dissipation (Figure 4) possibly attributable to the transition with increasing frequency between the saturated-isobaric and saturatedisolated regimes. This interpretation is broadly consistent with the results of the flexural and torsional oscillation tests on water-saturated specimens at low differential pressure. As expected in theory (e.g., O'Connell \& Budiansky, 1977), and as similarly observed in Li et al., 2018, the ultrasonic measurement at 1 MHz, and low-frequency water-saturated tests, reveal systematic stiffening of the shear modulus suggestive of saturated-isolated conditions (Figure 7).

\subsubsection{Bulk Modulus Dispersion and Dissipation at 2.5 MPa: The Draining Transition}

The results of the confining pressure oscillation show marked dispersion of $K$ and strongly associated dissipation only at 2.5 MPa water saturated (Figure 9). The dispersion for the water-saturated condition and associated dissipation are best explained in terms of draining, that is, stress-induced fluid flow between the specimen and the external reservoir. (e.g., Dunn, 1987; Gardner, 1962; Morig \& Burkhardt, 1989).

To gain further insight into this behavior, the Biot-Gassmann model can be used to relate the drained bulk modulus to the undrained low-frequency bulk modulus, obtained from the experiment. Taking $K_{\mathrm{d}}$ as $8 \mathrm{GPa}$, $K_{\mathrm{m}}$ as $44 \mathrm{GPa}$ (uncracked glass, Li, 2016), $\Phi_{c}=0.0074$, and $K_{\mathrm{f}}=2.25 \mathrm{GPa}$, the undrained bulk modulus from Gassmann's prediction is obtained as $39 \mathrm{GPa}$. The value of bulk modulus for the water-saturated condition at the lowest measurement frequency $(0.004 \mathrm{~Hz}, 32 \mathrm{GPa})$ is lower than this estimate-suggesting that the BiotGassmann model suitably describes the ongoing bulk stiffening occurring with suppression of draining.

Fully undrained behavior with $K_{\mathrm{u}} \sim 49 \mathrm{GPa}$ is seen only at frequencies $>0.02 \mathrm{~Hz}$. In addition, the ultrasonic bulk modulus for the water-saturated conditions is broadly comparable to the bulk modulus at $1 \mathrm{~Hz}-$ 


\section{Hydrostatic pressure}

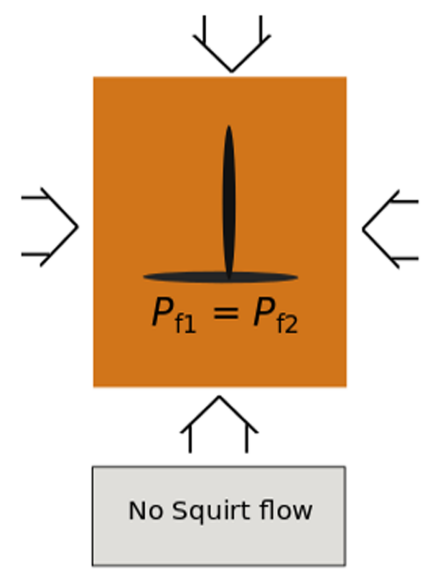

Pure Shear stress

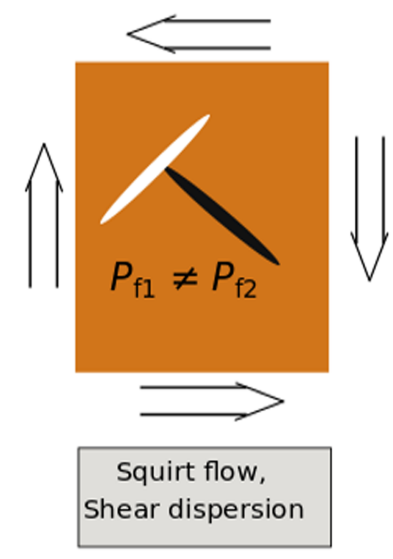

Figure 10. Effective elastic response for a medium containing only cracks, of random orientation with a given aspect ratio $\alpha$, under hydrostatic compression (left) and shear stress (right). $P_{\mathrm{f} 1}$ and $P_{\mathrm{f} 2}$ are the pore fluid pressures in representative cracks with diverse orientation relative to the applied stress. Bulk modulus for the saturated isobaric (undrained) regime will be same as that for saturated isolated regime, whereas the difference in pore fluid pressure in the different cracks will results in shear modulus dispersion between the saturated isobaric and saturated isolated regimes. Observed in shear modulus. (after Jackson, 1991, according to the model of O'Connell \& Budiansky, 1977; c.f., Adelinet et al., 2011; Li et al., 2018). implying the absence of significant dispersion for $0.02 \mathrm{~Hz}<f<0.5 \mathrm{MHz}$ (Figure 9). This observation is consistent with the expectation (Figure 10) that the bulk modulus will be unaffected by the transition between saturated-isobaric and saturated-isolated regimes in a medium containing a narrow distribution of crack aspect ratios (O'Connell \& Budiansky, 1977). For a cracked medium with a unique crack aspect ratio under externally imposed hydrostatic stress, all cracks will be equally pressurized, and accordingly, there will exist no stress-induced gradients in pore fluid pressure to drive squirt flow. (Figure 10).

Furthermore, the standard anelastic model (Nowick \& Berry, 1972) or Zener viscoelastic model (e.g., Mavko et al., 2009) can be used to describe the frequency-dependent coupling of the observed dispersion and dissipation across the draining transition. The model parameters are the unrelaxed $\left(K_{\mathrm{u}}=49 \mathrm{GPa}\right)$ and relaxed $\left(K_{\mathrm{d}}=8 \mathrm{GPa}\right)$ moduli and the characteristic frequency $(0.002 \mathrm{~Hz})$, which can be estimated from the experimental data. The Zener model evidently provides a broadly satisfactory fit for the dispersion and dissipation marking the transition with increasing frequency between the drained and saturated-isobaric regimes (Figure 9). Such satisfactory fit implies only one viscoelastic mechanism is at play. Thus, the draining transition is adequately modeled with a unique characteristic frequency.

Relating the dispersion to the characteristic frequencies for fluid flow can provide further relevant information. For the FDL2 cracked specimen, permeability (at $P_{\mathrm{d}}=2.5 \mathrm{MPa}$ ) $\sim 3 \times 10^{-19} \mathrm{~m}^{2}$ and $l$ the diffusion length (i.e. half of the specimen's length) is $0.034 \mathrm{~m}$. Shear modulus and Poisson's ratio of the frame is given as $31 \mathrm{GPa}$ and 0.21 , respectively (Li et al., 2018). From the pressure dependence of the wave speed which indicates crack closure at $\sim 15 \mathrm{MPa}$, representative aspect ratio (obtained using $P_{\mathrm{c}}=E \alpha$ ) is $\leqslant 2 \times 10^{-4}$. On the basis of equation (9), the characteristic frequency for drainage $f_{\mathrm{dr}}(\sim 10 \mathrm{mHz})$ is broadly consistent with the observations. Alternatively, a crack porosity near 0.0032 is inferred by requiring that equation (9) precisely yield the observed draining frequency $f_{\mathrm{dr}}(\sim 2 \mathrm{mHz})$. This is markedly less than the crack porosity at ambient conditions (0.0074)—consistent with other indications (Li et al., 2018) that much of the total porosity may consist of uniform partings closed by negligible pressure.

Compelling support for the occurrence of draining comes from the nonzero value of the pseudo-Skempton coefficient $\left(B^{*}\right)$ at low frequencies. The marked increase of $B^{*}$ with decreasing frequency, especially below $0.02 \mathrm{~Hz}$, provides unambiguous evidence of reversible fluid flow between the specimen and the external reservoir.

\subsubsection{Dispersion of Young's and Shear Moduli and Related Dissipation for $\boldsymbol{P}_{\mathrm{d}}<10 \mathrm{MPa}$ : The Squirt Transition}

The complementary techniques of axial and flexural stress oscillation were used to measure Young's modulus and dissipation most comprehensively on cracked specimens FDL2, and SAGR2, under both dry and fluid-saturated conditions. In general, the two techniques produced similar results for the water-saturated conditions in that they both show systematic frequency dependence of the measured Young's modulus. Results on water-saturated FDL2 specimen, tested under axial loading, show dispersion of Young's modulus at $P_{\mathrm{d}}=2.5-10 \mathrm{MPa}$, accompanied by different patterns of frequency-dependent dissipation.

The observed frequency dependence of the Young's modulus and associated dissipation at higher-thandraining frequencies is plausibly attributed to local (squirt) fluid flow. The marked increase in Young's modulus with increasing frequency for the water-saturated condition is attributed to an increasing shear modulus associated with suppression of squirt flow (through $1 / E=1 / 3 G+1 / 9 K$ ).

The Zener (standard linear solid) model can be used to describe the observed frequency dependence of the dissipation across the transition from the saturated-isobaric to the saturated-isolated regime. The observed strong dispersion, however, spans at least 3 orders of magnitude in frequency (Figure 11)—compared with only 1 order of magnitude for the Zener model (Figure 9). So a single peak Zener or single element Cole-Cole 

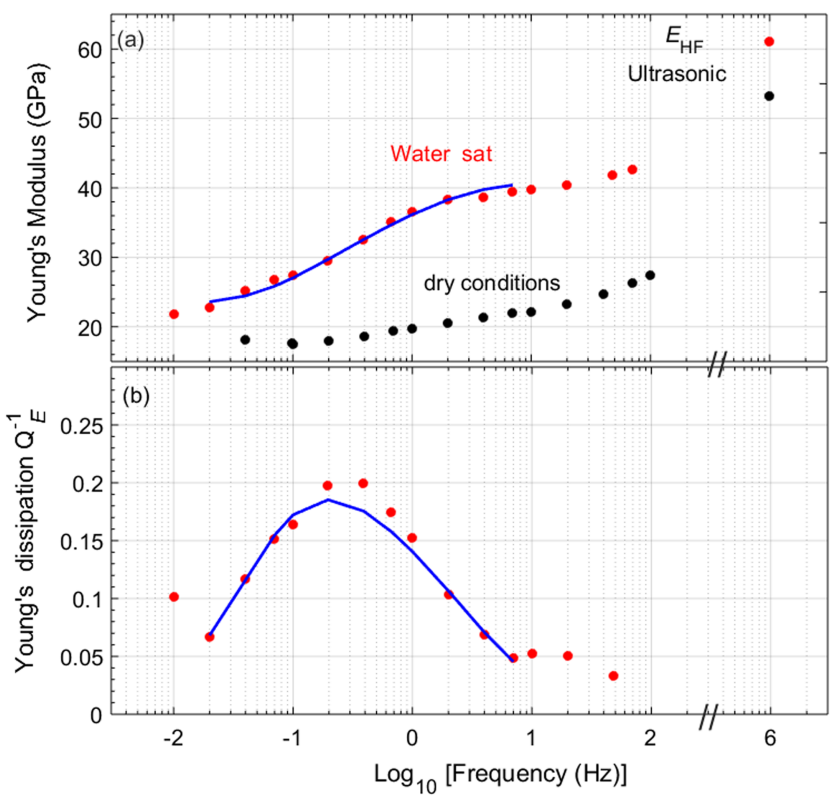

Figure 11. Frequency dependent Young's (a) modulus and (b) dissipation obtained from the axial forced oscillation test for the water-saturated FDL2 specimen. Red and black symbols indicate the data points, whereas the blue curve represent a modified Zener model extended to include a distribution of relaxation times, optimally fitted to Young's modulus and associated dissipation data pairs to match the observed dispersion and dissipation peak. Data at the lowest and the three highest frequencies were excluded. model (Cole \& Cole, 1941) cannot capture the observed broadband Young's dissipation-suggesting that a distribution of relaxation times is instead required to describe the observation.

In Figure 11, the Young's modulus and dissipation data for FDL2 are compared with a standard linear solid model extended to include a continuous distribution of relaxation times across a prescribed range. The model satisfactorily fits the observations-confirming that the observed Young's modulus dispersion and dissipation can be attributed to the squirt flow transition. That the transition is centered near $0.3 \mathrm{~Hz}$ rather than the value of $55 \mathrm{~Hz}$ estimated from equation (10) requires a distribution of generally somewhat lower aspect ratios and/or a higher effective viscosity resulting from chemical interaction between the water and the siliceous surfaces of the narrow cracks.

Further support for this interpretation can be derived from the shear modulus dispersion at low $P_{\mathrm{d}}<10 \mathrm{MPa}$ observed for the smaller-diameter specimens tested with the ANU attenuation apparatus. For specimen SAGR2, which has been more intensively probed within the realm of low differential pressure than the other specimens, significant dispersion of the shear modulus and associated dissipation are evident at $0.01-1 \mathrm{~Hz}$, most clearly at the lowest differential pressure of $5 \mathrm{MPa}$. Another indication of squirt flow is the negative frequency dependence of Poisson's ratio, measured during oscillation of the axial stress (Figure 4). The fluid insensitivity of the bulk modulus for a medium with a single crack aspect ratio across the transition between the saturated-isobaric to saturated-isolated regimes implies that the expected increase in shear and hence Young's modulus must lead to a decrease in Poisson's ratio-consistent with the behavior observed with FDL2 in Figure 4 (cf. Pimienta et al., 2016).

Higher Young's moduli, both dry and water saturated, at megahertz frequency relative to those at lower frequencies, may be best explained as a path effect. For a cracked specimen of this kind, it is possible that the first-arriving part of the elastic wave preferentially samples a largely crack-free and therefore fast path, according to Fermat's principle. The relatively wide spacing and preferred orientation of cracks (Figure 1) supports the suggestion that much of the dispersion, observed between $200 \mathrm{~Hz}$ of the low-frequency data and the ultrasonic data, could be path related.

\section{Conclusions}

Both permeability and seismic properties have been measured with complementary techniques on a suite of thermally cracked glass specimens. Whereas the ANU apparatus allows low-frequency measurement of the Young's and shear moduli, the ENSP set up permits low-frequency measurements of Poisson's ratio and the Young's and bulk moduli, through the oscillation of the axial stress and confining pressure, respectively. Ultrasonic wave speed measurements constrain the behaviors at $\mathrm{MHz}$ frequency including elastic anisotropy due to the thermal cracks.

Dry ultrasonic wave speeds, low-frequency moduli $(E, G$, and $K)$ and axial strain increase markedly with increasing pressure below $15 \mathrm{MPa}$, reflecting the closure of cracks with aspect ratios $<2 \times 10^{-4}$.

Permeability results show distinct pressure-dependent trends with different fluids. Argon and pentane permeability are broadly similar, consistently higher and more mildly pressure-dependent than for water. The unmeasurably low water permeability at differential pressures $>10 \mathrm{MPa}$ is interpreted to reflect chemical interaction between the polar water molecule and the siliceous surfaces of the very narrow cracks.

The observed dispersions (with associated dissipation) of the bulk and Young's moduli for the watersaturated specimen FDL2 at $P_{\mathrm{d}}=2.5 \mathrm{MPa}$ suggest that the draining transition is centered near $2 \mathrm{mHz}$, with a broad squirt flow transition centered near $0.3 \mathrm{~Hz}$. The effect of increasing $P_{\mathrm{d}}$ beyond $2.5 \mathrm{MPa}$ is to reduce crack aperture such that chemical interactions between the polar water molecule and the siliceous crack 
surfaces substantially increase the effective viscosity, thereby dramatically reducing the characteristic frequencies both for draining and squirt flow. A submillihertz squirt frequency for $P_{\mathrm{d}} \geq 10 \mathrm{MPa}$ is required to explain the observation of saturated-isolated conditions for the forced oscillation measurements on watersaturated specimens.

Our laboratory findings present new insight into the seismic properties of a fluid-saturated synthetic medium which contains only cracks with a narrow distribution of aspect ratios, rather than the mixture of cracks and pores typical of crustal rocks. Such findings not only further confirm the predictions of theoretical models (e.g., O'Connell \& Budiansky, 1977) but also highlight the need for care in the seismological application of laboratory ultrasonic data for fluid-saturated cracked media.

\section{Appendix A: Fitting the Multipath Ultrasonic Wave Speeds to a Model of Transverse Elastic Isotropy}

For $P_{\mathrm{c}}<15 \mathrm{MPa}$, the multipath wave speed vary systematically with propagation direction. Isotropic behavior, however, is observed $P_{\mathrm{c}}>15 \mathrm{MPa}$ with independent elastic constants $c_{11}=\rho V_{P}^{2}=85 \mathrm{GPa}$, and $c_{12}=$ $c_{11}-2 \rho V_{S}^{2}=22 \mathrm{GPa}$.

In this appendix, we describe in the following steps our approach for fitting the wave speeds data to a model of transverse isotropy with symmetry axis parallel with the axis of the cylindrical specimen.

A medium with transversely isotropic elasticity has five independent elastic stiffness components $\left(c_{11}, c_{12}, c_{13}\right.$, $\left.c_{33}, c_{44}\right)$ (e.g., Nye, 1985). So the stress-strain relationship becomes

$$
\left\{\begin{array}{l}
\sigma_{1} \\
\sigma_{2} \\
\sigma_{3} \\
\sigma_{4} \\
\sigma_{5} \\
\sigma_{6}
\end{array}\right\}=\left\{\begin{array}{cccccc}
c_{11} & c_{12} & c_{13} & 0 & 0 & 0 \\
c_{12} & c_{11} & c_{13} & 0 & 0 & 0 \\
c_{13} & c_{13} & c_{33} & 0 & 0 & 0 \\
0 & 0 & 0 & c_{44} & 0 & 0 \\
0 & 0 & 0 & 0 & c_{44} & 0 \\
0 & 0 & 0 & 0 & 0 & c_{66}
\end{array}\right\}\left\{\begin{array}{l}
\varepsilon_{1} \\
\varepsilon_{2} \\
\varepsilon_{3} \\
\varepsilon_{4} \\
\varepsilon_{5} \\
\varepsilon_{6}
\end{array}\right\}
$$

where

$$
\mathrm{c}_{66}=\frac{1}{2}\left(\mathrm{c}_{11}-\mathrm{c}_{12}\right)
$$

The quasi-P wave modulus is given as

$$
M_{Q P}(\theta)=\frac{1}{2}(N+\sqrt{M})
$$

with

$$
\begin{gathered}
\mathrm{N}=\mathrm{c}_{11} \sin ^{2} \theta+\mathrm{c}_{33} \cos ^{2} \theta+\mathrm{c}_{44} \\
\mathrm{M}=D^{2}+\left(c_{13}+c_{44}\right)^{2} \sin ^{2} 2 \theta \\
\mathrm{D}=\left(c_{11}-c_{44}\right) \sin ^{2} \theta-\left(c_{33}-c_{44}\right) \cos ^{2} \theta
\end{gathered}
$$

and

$$
M_{S H}(\theta)=\left(c_{66} \sin ^{2} \theta+c_{44} \cos ^{2} \theta\right)
$$

For small perturbations, the deviation from isotropic behavior as a linear combinations of perturbations can be written as 


$$
\delta M_{\mathrm{QP}}(\theta)=M_{\mathrm{QP}, \mathrm{obs}}(\theta)-M_{\mathrm{QP}, \text { iso }}(\theta)=f_{11} \delta \mathrm{c}_{11}+f_{33} \delta \mathrm{c}_{33}+f_{44} \delta \mathrm{c}_{44}+f_{13} \delta \mathrm{c}_{13}
$$

$\delta M_{\mathrm{QP}}(\theta)$ is the modulus perturbations or deficit from isotropic behavior, $M_{\mathrm{QP}, \mathrm{obs}}(\theta)$ is the quasi-P wave modulus calculated from the data at $P_{\mathrm{c}}<15 \mathrm{MPa}, M_{\mathrm{QP}}$,iso $(\theta)$ is the quasi-P wave modulus for the isotropic conditions (i.e., at $\left.P_{\mathrm{c}}>15\right)$, and $f_{\mathrm{ij}}(\theta)\left(=\partial \delta M_{\mathrm{QP}}(\theta) / \partial \mathrm{c}_{\mathrm{ij}}\right)$ is the sensitivities for the perturbations.

Accordingly, the partial derivatives or sensitivities $f_{\mathrm{ij}}=\partial \delta \mathrm{M}_{\mathrm{QP}}(\theta) / \partial \mathrm{c}_{\mathrm{ij}}$ are given by

$$
\begin{gathered}
f 11=\frac{1}{2}\left(1+\frac{D}{\sqrt{M}}\right) \sin ^{2} \theta \\
f 33=\frac{1}{2}\left(1-\frac{D}{\sqrt{M}}\right) \cos ^{2} \theta \\
f 44=\frac{1}{2}\left\{1+\left[D \cos 2 \theta+\left(c_{13}+c_{44}\right) \sin ^{2} 2 \theta\right] / \sqrt{M}\right\} \\
f 13=\frac{1}{2}\left[\left(c_{33}+c_{44}\right) / \sqrt{M}\right] \sin ^{2} 2 \theta
\end{gathered}
$$

In the isotropic reference state, these expressions reduce to

$$
\begin{gathered}
f 11=\frac{1}{2}(1-\cos 2 \theta) \sin ^{2} \theta=\sin 4 \theta \\
f 33=\frac{1}{2}(1+\cos 2 \theta) \cos ^{2} \theta=\cos 4 \theta \\
f 44=\sin ^{2} 2 \theta \\
f 13=\frac{1}{2} \sin ^{2} 2 \theta
\end{gathered}
$$

The corresponding quantities for $M_{\mathrm{SH}}(\theta)$ are

$$
\begin{gathered}
M_{S H}(\theta)=\rho v_{S H}^{2}=c_{44} \cos ^{2} \theta+\frac{1}{2}\left(c_{11}-c_{12}\right) \sin 2 \theta \\
f 11=\frac{1}{2} \sin ^{2} \theta \\
f 12=-\frac{1}{2} \sin ^{2} \theta \\
f_{44}=\cos ^{2} \theta
\end{gathered}
$$

We note that the sensitivities of $M_{\mathrm{QP}}$ to variation of $\mathrm{c}_{11}\left(\mathrm{c}_{33}\right)$ increase (decrease) monotonically and strongly with increasing $\theta$, whereas the sensitivities for variation of $\mathrm{c}_{44}$ and $\mathrm{c}_{13}$ are greatest at intermediate values of $\theta$. Moreover, for isotropic elasticity only, $f_{44} \equiv 2 f_{13}$. The existence of this relationship means that the perturbations in $\mathrm{c}_{44}$ and $\mathrm{c}_{13}$ are not separately resolvable, only the combination $\delta\left(2 \mathrm{c}_{44}+\mathrm{c}_{13}\right)=2 \delta \mathrm{c}_{44}+\delta \mathrm{c}_{13}$. Only the $\mathrm{SH}$ modulus has sensitivity to $\mathrm{c}_{12}$.

Next, the observed modulus deficits $\left(\delta M_{Q P}(\theta), \delta M_{S H}(\theta=90)\right)$ and the sensitivities $f_{i j}(\theta)$ were substituted into equation (A4) to yield a set of simultaneous linear equations that were solved for the perturbations $\delta \mathrm{c}_{\mathrm{ij}}$.

Finally, the perturbations, $\delta \mathrm{c}_{i j}$, obtained at each pressure are added to the isotropic $\mathrm{c}_{\mathrm{ij}}$ as follows, to obtain the estimated values of $\mathrm{c}_{\mathrm{ij}}$ within the transversely isotropic model: 


$$
\begin{gathered}
c_{11, \mathrm{VTI}}=c_{11, \mathrm{ISO}}+\delta c_{11} \\
c_{12, \mathrm{VTI}}=c_{12, \mathrm{ISO}}+\delta c_{12} \\
c_{33, \mathrm{VTI}}=c_{33, \mathrm{ISO}}+\delta c_{33}=c_{11, \mathrm{ISO}}+\delta c_{33} \\
\left(2 c_{44}+c_{13}\right)_{\mathrm{VTI}}=\left(2 c_{44}+c_{13}\right)_{\mathrm{ISO}}+\delta\left(2 c_{44}+c_{13}\right)=c_{11, \mathrm{ISO}}+\delta\left(2 c_{44}+c_{13}\right)
\end{gathered}
$$

For the unresolved relative magnitudes of $\delta c_{44}$ and $\delta c_{13}$, the best fit was obtained with setting $\delta c_{13}=0$, thereby attributing all of the perturbation $\delta\left(2 c_{44}+c_{13}\right)$ to $c_{44}$. So

$$
\begin{gathered}
c_{13, \mathrm{VTI}}=c_{13, \mathrm{ISO}}=c_{12, \mathrm{ISO}} \\
c_{44, \mathrm{VTI}}=\frac{1}{2}\left(c_{11}-c_{12}\right)_{\mathrm{ISO}}+\frac{1}{2} \delta\left(2 c_{44}+c_{13}\right)
\end{gathered}
$$

\section{Appendix B: Elastic Moduli for Oscillation of Confining Pressure and Axial Stress}

\section{B.1. Introduction}

In this appendix, we provide an analysis of the response of a transversely isotropic medium for the deformation under hydrostatic and axial loading, thereby obtaining expressions for the measured bulk $K$ and Young's $E$ moduli and Poisson's ratio $\nu$. We show that $E$ and $\nu$ measured by oscillation of the axial stress can be combined for direct comparison with the bulk modulus, obtained through oscillation of hydrostatic pressure, only for an isotropic medium.

\section{B.2. The Elastic Moduli}

B.2.1. For axial loading condition $\left(\sigma_{i}=\sigma \delta_{i 3}\right)$, equation (A1) can be solved to obtain the strains $\varepsilon_{i}$ as

$$
\begin{gathered}
\varepsilon_{1}=\varepsilon_{2}=\frac{-\sigma c_{13}}{c_{33}\left(c_{11}+c_{12}\right)-2 c_{13}^{2}} ; \\
\varepsilon_{3}=\frac{-\sigma\left(c_{11}+c_{12}\right)}{c_{33}\left(c_{11}+c_{12}\right)-2 c_{13}^{2}} ; \\
\varepsilon_{4}=\varepsilon_{5}=\varepsilon_{6}=0
\end{gathered}
$$

Thus, the axial Young's modulus, $E$, defined as $\sigma / \varepsilon_{3}$ then becomes

$$
E=c_{33}-\frac{2 c_{13}^{2}}{c_{11}+c_{12}}
$$

and Poisson's ratio $\nu\left(=-\varepsilon_{1} / \varepsilon_{3}\right)$ is expressed as

$$
=\frac{c_{13}}{c_{11}+c_{12}}
$$


B.2.2. Under hydrostatic loading conditions $\left[\sigma_{i}=-P\left(\delta_{i 1}+\delta_{i 2}+\delta_{i 3}\right)\right]$ we obtain the following strains, $\varepsilon_{1}$ and $\varepsilon_{3}$ required for volumetric strain $\left(\varepsilon_{\mathrm{vol}}=2 \varepsilon_{1}+\varepsilon_{3}\right)$ as below

$$
\begin{gathered}
\varepsilon_{1}=\varepsilon_{2}=-P \frac{c_{33}-c_{13}}{c_{33}\left(c_{11}+c_{12}\right)-2 c_{13}^{2}} ; \\
\varepsilon_{3}=-P \frac{c_{11+} c_{12}-2 c_{13}}{c_{33}\left(c_{11}+c_{12}\right)-2 c_{13}^{2}} ;
\end{gathered}
$$

So $\varepsilon_{\mathrm{vol}}$ or $d V / V$ becomes

$$
-P \frac{2 c_{33}+c_{11}+c_{12}-4 c_{13}}{c_{33}\left(c_{11}+c_{12}\right)-2 c_{13}^{2}}
$$

The foregoing analysis shows that the effective moduli of a VTI medium obtained

1. Through the oscillation of the axial stress as

$$
E=c_{33}-\frac{2 c_{13}^{2}}{c_{11}+c_{12}} ; \nu=-\frac{c_{13}}{c_{11}+c_{12}}
$$

2. Through the oscillation of the hydrostatic pressure as

$$
K_{c p}=-P /\left(\frac{d V}{V}\right)=\frac{c_{33}\left(c_{11}+c_{12}\right)-2 c_{13}^{2}}{2 c_{33}+c_{11}+c_{12}-4 c_{13}} .
$$

Combining $E$ and $\nu$ as measured in axial oscillation for a transversely isotropic material, but with the assumption of isotropic elasticity (where $K=\frac{E}{3(1-2 v)}$ ), leads to

$$
K_{a x}=\frac{c_{33}\left(c_{11}+c_{12}\right)-2 c_{13}^{2}}{3\left[c_{11}+c_{12}-2 c_{13}\right]} ; \neq K_{c p} \text { above. }
$$

\section{Acknowledgments}

The authors gratefully acknowledge technical assistance at ANU by Hayden Miller. First author acknowledges $\mathrm{PhD}$ scholarship support from the Australian National University and also thanks Kathryn Hayward (at ANU) for help with polished sections. We also thank Nicolas Aurélien (at ENS) for helping with multipath ultrasonic measurements. Finally, the authors are delighted to contribute to the special volume in honor of J. B. Walsh - a giant who has left an enduring mark on the field of rock physics. The laboratory data supporting this paper has been archived by the ANU Data Commons and can be found online (at https://dx. doi.org/10.25911/5e1e43277ef68).

\section{References}

Adam, L., Batzle, M., Lewallen, K. T., \& Van Wijk, K. (2009). Seismic wave attenuation in carbonates. Journal of Geophysical Research, 114, B06208. https://doi.org/10.1029/2008JB005890

Adam, L., Batzle, M., \& Brevik, I. (2006). Gassmann's fluid substitution and shear modulus variability in carbonates at laboratory seismic and ultrasonic frequencies. Geophyscis, 71(6), F173-F183. Retrieved from. https://doi.org/10.1190/1.2358494

Adelinet, M., Fortin, J., \& Guéguen, Y. (2011). Dispersion of elastic moduli in a porous-cracked rock: Theoretical predictions for squirtflow. Tectonophysics, 503(1-2), 173-181. https://doi.org/10.1016/j.tecto.2010.10.012

Adelinet, M., Fortin, J., Guéguen, Y., Schubnel, A., \& Geoffroy, L. (2010). Frequency and fluid effects on elastic properties of basalt: Experimental investigations. Geophysical Research Letters, 37, L02303. https://doi.org/10.1029/2009GL041660

Audonnet, F., \& Pádua, A. A. H. (2001). Simultaneous measurement of density and viscosity of n-pentane from 298 to 383 K and up to 100 MPa using a vibrating-wire instrument. Fluid Phase Equilibria, 181(1-2), 147-161. https://doi.org/10.1016/S0378-3812(01)00487-3

Batzle, M. L., Han, D.-H., \& Hofmann, R. (2006). Fluid mobility and frequency-dependent seismic velocity-Direct measurements. Geophysics, 71(1), N1. https://doi.org/10.1190/1.2159053

Biot, M. A. (1956a). Theory of propagation of elastic waves in a fluid-saturated porous solid. The Journal of the Acoustical Society of America, 28(2), 13. https://doi.org/10.1121/1.1908239

Biot, M. A. (1956b). Theory of propagation of elastic waves in a fluid saturated porous solid. II. Higher frequency range. The Journal of the Acoustical Society of America, 28(2), 179-191. https://doi.org/10.1121/1.1908241

Birch, F. (1961). The velocity of compressional waves in rocks to 10 kilobars: 2. Journal of Geophysical Research, 66(7), 2199-2224. https:// doi.org/10.1029/JZ066i007p02199

Borgomano, J. V. M., Pimienta, L., Fortin, J., \& Guéguen, Y. (2017). Dispersion and attenuation measurements of the elastic moduli of a dual-porosity limestone. Journal of Geophysical Research - Solid Earth, 122, 2690-2711. https://doi.org/10.1002/2016JB013816

Bourbié, T., Coussy, O., \& Zinszner, B. (1987). Acoustics of porous media. Paris: Editions Technip.

Brace, W. F., Walsh, J. B., \& Frangos, W. T. (1968). Permeability of granite under high pressure. Journal of Geophysical Research, 73(6), 2225-2236. https://doi.org/10.1029/JB073i006p02225

Chapman, S., Borgomano, J. V. M., Yin, H., Fortin, J., \& Quintal, B. (2018). Forced oscillation measurements of seismic wave attenuation and stiffness moduli dispersion in glycerine-saturated Berea sandstone. Geophysical Prospecting, 1-13. https://doi.org/10.1111/13652478.12710

Chapman, S., Quintal, B., Holliger, K., Baumgartner, L., \& Tisato, N. (2018). Laboratory measurements of seismic attenuation and Young's modulus dispersion in a partially and fully water-saturated porous sample made of sintered borosilicate glass. Geophysical Prospecting, 66(7), 1384-1401. https://doi.org/10.1111/1365-2478.12643 
Clark, V. A., Tittmann, B. R., \& Spencer, T. W. (1980). Effect of volatiles on attenuation (Q - 1) and velocity in sedimentary rocks. Journal of Geophysical Research, 85(B10), 5190. https://doi.org/10.1029/JB085iB10p05190

Cleary, M. P. (1978). Elastic and dynamic response regimes of fluid-impregnated solids with diverse microstructures. International Journal of Solids and Structures, 14(10), 795-819. https://doi.org/10.1016/0020-7683(78)90072-0

Cline, C. J., \& Jackson, I. (2016). Relaxation of the bulk modulus in partially molten dunite? Geophysical Research Letters, 43(22), 11,644-11,651. https://doi.org/10.1002/2016GL071004

Cole, K. S., \& Cole, R. H. (1941). Dispersion and absorption in dielectrics I. Alternating current characteristics. The Journal of Chemical Physics, 9(4), 341-351. https://doi.org/10.1063/1.1750906

David, E. C., Fortin, J., Schubnel, A., Guéguen, Y., \& Zimmerman, R. W. (2013). Laboratory measurements of low- and high-frequency elastic moduli in Fontainebleau sandstone. Geophysics, 78(5), D369-D379. https://doi.org/10.1190/geo2013-0070.1

Ding, Z. S., Alliez, J., Boned, C., \& Xans, P. (1997). Automation of an ultrasound velocity measurement system in high-pressure liquids. Measurement Science and Technology, 8, 154-161. https://doi.org/10.1088/0957-0233/8/2/007

Dunn, K. (1987). Sample boundary effect in acoustic attenuation of fluid-saturated porous cylinders. The Journal of the Acoustical Society of America, 81(5), 1259-1266. https://doi.org/10.1121/1.394529

Dvorkin, J., Mavko, G., \& Nur, A. (1995). Squirt flow in fully saturated rocks. Geophysics, 60(1), 97-107. https://doi.org/10.1190/ 1.1443767

Gardner, G. H. F. (1962). Extensional waves in fluid-saturated porous cylinders. The Journal of the Acoustical Society of America, 34(1), 36-40. https://doi.org/10.1121/1.1909010

Gassmann, F. (1951). Über die elastizität poröser medien. Vierteljahrsschrift Der Naturforschenden Gesellschaft in Zurich, 96, 1-23.

Guéguen, Y., \& Kachanov, M. (2011). Effective elastic properties of cracked rocks-An overview. Mechanics of Crustal Rocks, 73-125. https://doi.org/10.1007/978-3-7091-0939-7

Guéguen, Y., \& Palciauskas, V. (1994). Introduction to the physics of rocks. Princeton, NJ: Princeton University Press.

Heap, M. J., Reuschlé, T., Farquharson, J. I., \& Baud, P. (2018). Permeability of volcanic rocks to gas and water. Journal of Volcanology and Geothermal Research, 354, 29-38. https://doi.org/10.1016/j.jvolgeores.2018.02.002

Jackson, I. (1991). The petrophysical basis for the interpretation of seismological models for the continental lithosphere. In B. J. Drummond (Eds), Geological Society of Australia Special Publication (Vol. 17, pp. 81-114). Sydney, NSW, Australia.

Jackson, I., \& Paterson, M. S. (1993). A high-pressure, high-temperature apparatus for studies of seismic wave dispersion and attenuation. Pure and Applied Geophysics, 141(2-4), 445-466. https://doi.org/10.1007/BF00998339

Jackson, I., Schijns, H., Schmitt, D. R., Mu, J., \& Delmenico, A. (2011). A versatile facility for laboratory studies of viscoelastic and poroelastic behaviour of rocks. The Review of Scientific Instruments. https://doi.org/10.1063/1.3592154

Jech, J., \& Pšenčík, I. (1989). First-order perturbation method for anisotropic media. Geophysical Journal International, 99(2), 369-376 https://doi.org/10.1111/j.1365-246X.1989.tb01694.x

Khazanehdari, J., \& Sothcott, J. (2003). Variation in dynamic elastic shear modulus of sandstone upon fluid saturation and substitution. Geophysics, 68(2), 472-481. https://doi.org/10.1190/1.1567213

Klinkenberg, L. J. (1941). The permeability of porous media to liquids and gases. In Drilling and Production Practice (pp. 200-213). New York: American Petroleum Institute.

Li, Y. (2016). A broadband laboratory study of the seismic properties of cracked and fluid-saturated synthetic glass materials. PhD Thesis, Australian National University.

Li, Y., David, E. C., Nakagawa, S., Kneafsey, T. J., Schmitt, D. R., \& Jackson, I. (2018). A broadband laboratory study of the seismic properties of cracked and fluid-saturated synthetic glass media. Journal of Geophysical Research - Solid Earth, 123, 3501-3538. https://doi.org/ 10.1029/2017JB014671

Li, Y., Olin, M., David, E. C., Jackson, I., Schijns, H., \& Schmitt, D. R. (2014). Broadband laboratory measurements of dispersion in thermally cracked and fluid-saturated quartzite and a synthetic analogue. The Leading Edge, 33(6), 624-632. https://doi.org/10.1190/ tle33060624.1

Lu, C., \& Jackson, I. (1996). Seismic-frequency laboratory measurements of shear mode viscoelasticity in crustal rocks. I: Competition between cracking and plastic flow in thermally cycled Carrara marble. Physics of the Earth and Planetary Interiors, 94(1-2), 105-119. https://doi.org/10.1016/0031-9201(95)03079-4

Mallet, C., Fortin, J., Guéguen, Y., \& Bouyer, F. (2013). Effective elastic properties of cracked solids: An experimental investigation. International Journal of Fracture, 182(2), 275-282. https://doi.org/10.1007/s10704-013-9855-y

Mavko, G. M., \& Nur, A. (1979). Wave attenuation in partially saturated rocks. Geophysics, 2(2), 161-178.

Mavko, G., \& Jizba, D. (1991). Estimating grain-scale fluid effects on velocity dispersion in rocks. Geophysics, 56(12), 1940-1949. https://doi. org/10.1190/1.1443005

Mavko, G., Mukerji, T., \& Dvorkin, J. (2009). The rock physics handbook: Tools for seismic analysis of porous media, (2nd ed.). Cambridge: Cambridge University Press. https://doi.org/10.1017/CBO9780511626753

Mavko, G., \& Nur, A. (1975). Melt squirt in the asthenosphere. Journal of Geophysical Research, 80(11), 1444-1448. https://doi.org/10.1029/ JB080i011p01444

Mensch, T., \& Rasolofosaon, P. (1997). Elastic-wave velocities in anisotropic media of arbitrary symmetry-generalization of Thomsen's parameters $\varepsilon, \delta$ and $\gamma$. Geophysical Journal International, 128(1), 43-64. https://doi.org/10.1111/j.1365-246X.1997.tb04070.x

Mikhaltsevitch, V., Lebedev, M., \& Gurevich, B. (2014). A laboratory study of low-frequency wave dispersion and attenuation in watersaturated sandstones. The Leading Edge, 33(6), 616-622.

Morig, R., \& Burkhardt, H. (1989). Experimental evidence of Biot-Gardner theory. Geophysics, 54(4).

Murphy, W. F., Winkler, K. W., \& Kleinberg, R. L. (1986). Acoustic relaxation in sedimentary rocks: Dependence on grain contacts and fluid saturation. Geophysics, 51(3), 757-766. https://doi.org/10.1190/1.1442128

Nowick, A. S., \& Berry, B. S. (1972). Anelastic relaxation in crystalline solids (Vol. 11)

Nye, J. F. (1985). Physical properties of crystals: Their representation by tensors and matrices. Oxford: Clarendon Press: Oxford University Press.

O'Connell, R. J., \& Budiansky, B. (1977). Viscoelastic properties of fluid-saturated cracked solids. Journal of Geophysical Research, 82(36), 5719-5735. https://doi.org/10.1029/JB082i036p05719

Ohanian, V., Snyder, T. M., \& Carcione, J. M. (2006). Weak elastic anisotropy by perturbation theory. Geophysics, 71(3), D45-D58. https:// doi.org/10.1190/1.2194520

Ougier-Simonin, A., Guéguen, Y., Fortin, J., Schubnel, A., \& Bouyer, F. (2011). Permeability and elastic properties of cracked glass under pressure. Journal of Geophysical Research, 116, B07203. https://doi.org/10.1029/2010JB008077 
Pimienta, L., Fortin, J., \& Guéguen, Y. (2015). Bulk modulus dispersion and attenuation in sandstones. Geophysics, 80(2), D111-D127. https://doi.org/10.1190/geo2014-0335.1

Pimienta, L., Borgomano, J. V. M., Fortin, J., \& Guéguen, Y. (2017). Elastic dispersion and attenuation in fully saturated sandstones: Role of mineral content, porosity, and pressures. Journal of Geophysical Research - Solid Earth, 122(12), 9950-9965. https://doi.org/10.1002/ 2017JB014645

Pimienta, L., Fortin, J., \& Guéguen, Y. (2016). Effect of fluids and frequencies on Poisson's ratio of sandstone samples. Geophysics, 81(2), D35-D47. https://doi.org/10.1190/GEO-2015-0310.1

Sarout, J., Cazes, E., Delle Piane, C., Arena, A., \& Esteban, L. (2017). Stress-dependent permeability and wave dispersion in tight cracked rocks: Experimental validation of simple effective medium models. Journal of Geophysical Research - Solid Earth, 122(8), 6180-6201. https://doi.org/10.1002/2017JB014147

Sayers, C. M., \& Kachanov, M. (1995). Microcrack-induced elastic wave anisotropy of brittle rocks. Journal of Geophysical Research, 100(B3), 4149-4156. https://doi.org/10.1029/94JB03134

Schijns, H., Jackson, I., \& Schmitt, D. R. (2018). Shear modulus dispersion in cracked and fluid-saturated quartzites: Experimental observations and modeling. Journal of Geophysical Research - Solid Earth, 123, 2825-2840. https://doi.org/10.1002/ 2017JB014633

Song, L.-P., \& Koch, M. (2002). Anisotropic reference media and the possible linearized approximations for phase velocities of qS waves in weakly anisotropic media. Journal of Physics D: Applied, 35). Retrieved from. https://iopscience.iop.org/article/10.1088/0022-3727/35/ $22 / 316 /$ pdf

Spencer, J. W. (1981). Stress relaxations at low frequencies in fluid-saturated rocks: Attenuation and modulus dispersion. Journal of Geophysical Research, 86(B3), 1803-1812. https://doi.org/10.1029/JB086iB03p01803

Stewart, R. B., \& Jacobsen, R. T. (1989). Thermodynamic properties of argon from the triple point to $1200 \mathrm{~K}$ with pressures to $1000 \mathrm{MPa}$ Journal of Physical and Chemical Reference Data, 18(2).

Tittmann, B. R., Clark, V. A., Richardson, J. M., \& Spencer, T. W. (1980). Possible mechanism for seismic attenuation in rocks containing small amounts of volatiles. Journal of Geophysical Research, 85(B10), 5199. https://doi.org/10.1029/ JB085iB10p05199

Tutuncu, A. N., \& Sharma, M. M. (1992). The influence of fluids on grain contact stiffness and frame moduli in sedimentary rocks. Geophysics, 57(12), 1571-1582. https://doi.org/10.1190/1.1443225

Venard, J. K., \& Street, R. L. (1975). Elementary fluid mechanics, (5th ed.). New York: Wiley.

Vidal, D., Tufeu, R., Garrabos, Y., \& Le Neindre, B. (1979). Thermophysical properties of noble gases at room temperature up to 1 GPa. In B. Vodar, \& P. Marteau (Eds.), High Pressure Science and Technologies, Proceedings of the 7th International AIRAPT Conference (Vol 2, pp. 692-698). Oxford, New York: Pergamon Press.

Walsh, J. B. (1965). The effect of cracks on the compressibility of rock. Journal of Geophysical Research, 70(2), 381-389. https://doi.org/ 10.1029/JZ070i002p00381

Winkler, K. W., \& Nur, A. (1982). Seismic attenuation: Effects of pore fluids and frictional sliding. Geophysics, 47(1), 1-15. https://doi.org/ $10.1190 / 1.1441276$

Wong, T. F., \& Baud, P. (2008). Mechanical compaction of porous sandstone. Oil \& Gas Science and Technology, 63(1), 9-19. https://doi.org/ $10.2516 /$ ogst

Wu, G., Zhao, X., Tang, J., \& Du, Z. (2017). First-order perturbation approximation for rock elastic moduli in transversely isotropic media Science China Earth Sciences, 60(9), 1645-1654. https://doi.org/10.1007/s11430-016-9064-8

Yin, H., Borgomano, J. V. M., Wang, S., Tiennot, M., \& Guéguen, Y. (2019). Fluid substitution and shear weakening in clay-bearing sandstone at seismic frequencies. Journal of Geophysical Research - Solid Earth, 124, 1254-1272. https://doi.org/10.1029/2018JB016241

Zimmerman, R. W. (1991). Compressibility of sandstones. Amsterdam: New York: New York, NY, USA: Elsevier; Distributors for the United States and Canada, Elsevier Science Pub. Co.

Zoback, M. D., \& Byerlee, J. D. (1975). The effect of microcrack dilatancy on the permeability of Westerly granite. Journal of Geophysical Research, 80(5), 752-755. https://doi.org/10.1029/JB083iB09p04451 